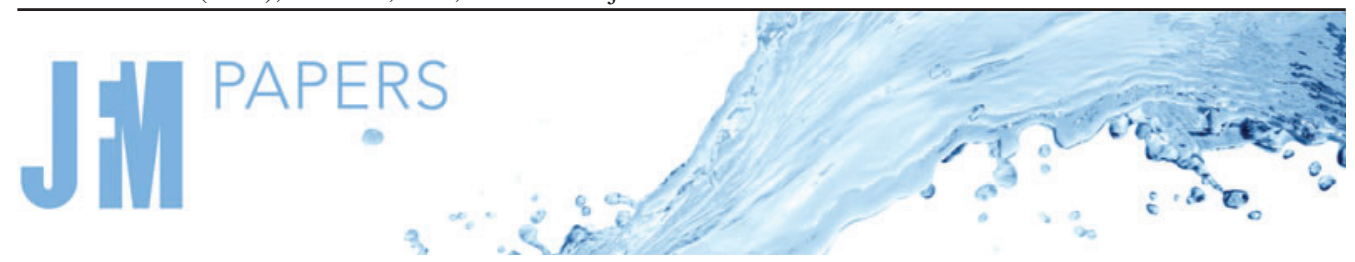

\title{
An asymptotic theory for the high-Reynolds-number flow past a shear-free circular cylinder
}

\author{
Anuj Kumar ${ }^{1}{ }^{\dagger}$, Nidhil M.A. Rehman ${ }^{1}$, Pritam Giri $^{1}$ and \\ Ratnesh K. Shukla ${ }^{1, \dagger}$ \\ ${ }^{1}$ Department of Mechanical Engineering, Indian Institute of Science, Bangalore, 560012, India
}

(Received 15 November 2020; revised 31 March 2021; accepted 7 May 2021)

We present an asymptotic theory for analytical characterization of the high-Reynoldsnumber incompressible flow of a Newtonian fluid past a shear-free circular cylinder. The viscosity-induced modifications to this flow are localized and except in the neighbourhood of the rear stagnation point, behave like a linear perturbation of the inviscid flow. Our theory gives a highly accurate description of these modifications by including the contribution from the most significant viscous term in a correctional perturbation expansion about an inviscid base state. We derive the boundary layer equation for the flow and deduce a similarity transformation that leads to a set of infinite, shear-free-condition-incompatible, self-similar solutions. By suitably combining members from this set, we construct an all-boundary-condition-compatible solution to the boundary layer equation. We derive the governing equation for vorticity transport through the narrow wake region and determine its closed-form solution. The near and far-field forms of our wake solution are desirably consistent with the boundary layer solution and the well-known, self-similar planar wake solution, respectively. We analyse the flow in the rear stagnation region by formulating an elliptic partial integro-differential equation for the distortion streamfunction that specifically accounts for the fully nonlinear and inviscid dynamics of the viscous correctional terms. The drag force and its atypical logarithmic dependence on Reynolds number, deduced from our matched asymptotic analysis, are in remarkable agreement with the high-resolution simulation results. The logarithmic dependence gives rise to a critical Reynolds number below which the viscous correction term, counterintuitively, reduces the net dissipation in the flow field.

Key words: boundary layer structure, wakes

\footnotetext{
$\dagger$ Email address for correspondence: ratnesh@iisc.ac.in

$\$$ Present address: Department of Applied Mathematics, Baskin School of Engineering,

University of California, Santa Cruz, CA 95064, USA
} 


\section{Introduction}

Fluid flows over sheer-free surfaces are radically distinct from those over which the relative motion between the fluid and the adjoining surface is forbidden by a no-slip condition. A shear-free boundary offers no resistance to the motion of the fluid along the surface, thus allowing the fluid to slip perfectly over it. This perfect slip condition that prevails over a shear-free surface has far-reaching consequences. Notably, the finite slippage of the fluid over a shear-free boundary suppresses vorticity generation from it, which in turn inhibits the boundary layer formation and growth processes (Leal 1989). The likelihood of flow separation on a shear-free boundary is therefore diminished substantially (Leal 1989; Legendre, Lauga \& Magnaudet 2009). As a consequence, surface stresses and hydrodynamic loads are drastically reduced. This exceptional feature of a shear-free surface is targeted in devising patterned superhydrophobic surfaces that effectively reduce drag by inducing significant slip over underwater bodies (Ou, Perot \& Rothstein 2004; You \& Moin 2007; Rothstein 2010; Bocquet \& Lauga 2011; Muralidhar et al. 2011; Karatay et al. 2013). The reduction in vorticity generation and flow separation offers additional advantages such as slip-enhanced transport (Haase et al. 2015; Haase \& Lammertink 2016; Rehman, Kumar \& Shukla 2017) and slip-induced flow stabilization (Legendre et al. 2009; Muralidhar et al. 2011; Seo \& Song 2012; Li et al. 2014; Xiong \& Yang 2017; Sooraj et al. 2020) as well. Advances in theoretical analysis and fundamental understanding of flow past shear-free surfaces is of significant technological importance and paramount for an effective realization of the full range of their drag and dissipation reducing, and transport enhancing capabilities.

In this work we develop an analytical model for the high-Reynolds-number flow past a shear-free circular cylinder. The configuration we consider consists of a stationary cylindrical boundary over which slip is realized through a finite tangential surface velocity. This configuration is of significance due to its remarkable drag-reducing and dissipation-minimizing attributes (Shukla \& Arakeri 2013), and, is quite distinct from the one in which a shear-free interface separates two fluid components with contrasting viscosities. The no-slip variant of this prototypical bluff body configuration has been extensively investigated both experimentally and through detailed simulations (e.g. Strouhal 1878; von Kármán 1911; Williamson 1996). Presence of hydrodynamic slip over the cylinder surface has been shown to suppress flow separation and prominent unsteady flow features including the Reynolds-number-dependent two- and three-dimensional vortex shedding patterns (Legendre et al. 2009; Rehman et al. 2017). Specifically, over a perfectly slipping cylindrical surface, computational investigations in the low-Reynolds-number regime ( $R e \leq 800, R e$ being the Reynolds number) have revealed formation of a relatively weak unseparated boundary layer and an asymptotic saturation of the maximum surface vorticity towards a Reynolds number independent upper limit (Legendre et al. 2009).

Our present investigation is, in part, motivated by the need of an in depth insight into the peculiar characteristics of the flow past general non-planar shear-free surfaces that only an elaborate theoretical analysis can facilitate. A distinctive outcome of our analysis is the theoretical prediction of a non-uniformity (switch in the sign) in the contribution to the net dissipation from the first-order viscous correctional terms. This non-uniformity occurs well beyond the highest Reynolds number of $10^{3}$ investigated in the previous works cited above and its existence is fully supported by high-resolution direct numerical simulations (see $\S 4$ ). The non-uniformity in the contribution to dissipation is altogether absent in an axisymmetric configuration (Moore 1963) and its existence is important from the perspective of drag reduction. Specifically, the non-uniformity in 


\section{High-Reynolds-number flow past a shear-free cylinder}

the contribution to dissipation implies a contrast in the optimal drag-reducing tangential surface velocity in the low- and high-Reynolds-number regime with the shear-free- and potential-flow-enabling tangential surface velocities minimizing the effective drag over a circular cylinder in the $R e \rightarrow 0$ and $R e \rightarrow \infty$ limits, respectively. Our theoretical approach provides a detailed description of the flow over boundary layer and wake regions $(\S \S 3.1$ and 3.3, respectively) and, most importantly, reveals the non-trivial dynamics of the flow in the vicinity of the rear stagnation region ( $\$ 3.2)$.

Our analysis relies on an asymptotic expansion about an inviscid, irrotational base state that follows from the potential flow theory. This frictionless base state violates the shear-free boundary condition over a non-planar perfectly slipping surface at any finite Reynolds number. Crucially, the inviscid base state suffers from the well-known D'Alembert's paradox for not only cylindrical but any arbitrarily shaped perfectly slipping boundary. To enforce a shear-free condition on the perfectly slipping cylinder, we introduce corrections in the form of a series consisting of terms that diminish progressively with the Reynolds number. For the most significant first-order correction term in the asymptotic expansion, we derive the appropriate governing equations that are uniquely applicable in each of the distinct, yet interdependent, boundary layer, rear stagnation and wake regions of the flow field. We subsequently determine the interconnected explicit form of the most significant correction term in these regions. Furthermore, by determining the dissipation associated with the shear-free-condition-consistent and D'Alembert's-paradox-resolved flow field, we show that the second-order term in the asymptotic expansion of the drag coefficient exhibits an atypical logarithmic dependence on the Reynolds number.

The asymptotic approach adopted in our work belongs to the wider class of well-established perturbation methods (Van Dyke 1975; Hinch 1991). Perturbation techniques have been used with remarkable success in the analysis of a range of flows including boundary layers, wakes and jets (Batchelor 2000; Schlichting \& Gersten 2003; Leal 2007). Specifically, to examine the high-Reynolds-number boundary layer characteristics over a spherical shear-free surface, Moore (1963) developed an axisymmetric, asymptotic expansion about an inviscid base state that is given by potential flow theory. In arriving at a correction to the celebrated drag force expression of Levich (1949), Moore (1963) relied on a linearized asymptotic analysis of the rear stagnation and the wake regions in addition to the boundary layer analysis. Extensions of the analysis to an oblate ellipsoidal shear-free surface and a stationary spherical interface that separates two fluids with finite viscosity contrast have been considered in Moore (1965) and Harper \& Moore (1968), respectively. Owing to the relatively large viscosity contrast between water and air, the frequently encountered water-air interface is very nearly shear free. As such, a large body of theoretical work on flow past a shear-free spherical boundary under diverse conditions arose out of an interest in bubble and droplet dynamics. A comprehensive review of the early efforts on theoretical modelling of the hydrodynamics of spherical and slightly deformed, near-spherical bubbles in motion can be found in Harper (1972). Besides being of fundamental importance, explicit expressions of the hydrodynamic forces exerted on bubbles are particularly useful in theoretical and computational investigations on the collective dynamics of bubble swarms. Considerable theoretical and computational effort has therefore gone into the characterization of forces experienced by a bubble undergoing motion in laminar and turbulent flow regimes. For an exhaustive treatment of this topic, we refer the interested readers to reviews by Magnaudet \& Eames (2000) and Michaelides (2003).

Our analysis and the analytical tractability of our approach are facilitated by an effective linearization of the governing equations over the boundary layer and wake regions. As in the case of a spherical shear-free interface, this linearization and the resulting 


\section{A. Kumar, N.M.A. Rehman, P. Giri and R.K. Shukla}

simplifications are direct consequences of the formation of a relatively weak boundary layer over the shear-free surface. Despite this apparent similarity in the analysis, numerous crucial differences do arise between axisymmetric spherical configuration considered by Moore (1963) and the cylindrical configuration analysed in our work. Notably, unlike the axisymmetric spherical configuration, the similarity solutions to the boundary layer equation for the cylindrical configuration turn out to be incompatible with the shear-free boundary condition. This incompatibility has necessitated development of techniques which exploit the linearity of the boundary layer equation and enable expression of the solution in the form of an infinite series of distinct self-similar solutions. In addition, the absence of a vortex stretching/contraction mechanism in two dimensions results in substantial disparities between the stagnation region of the planar cylindrical and the axisymmetric spherical configurations. Specifically, the Reynolds number dependence of the size of the stagnation region differs markedly $\left(O\left(R e^{-1 / 4}\right)\right.$ in the case of a shear-free cylinder vs $O\left(R e^{-1 / 6}\right)$ for a shear-free sphere). Moreover, the perturbations in the stagnation region of a shear-free cylinder are comparable to the base inviscid state so that the assumptions that form the basis of a linearized analysis (small perturbations) are invalidated. This complication in the analysis of the stagnation region is altogether absent in a shear-free axisymmetric spherical configuration. Importantly, our analysis reveals a striking dissimilarity between the Reynolds number dependence of the drag coefficient associated with flow past a shear-free sphere and the one corresponding to flow past a shear-free cylinder. A logarithmic dependence on the Reynolds number in the case of a circular cylinder implies that above a critical Reynolds number, the dissipation associated with a shear-free circular boundary exceeds the one for the irrotational potential flow. In contrast, the far simpler dependence on the Reynolds number in the case of axisymmetric flow past a sphere implies that the dissipation associated with a shear-free spherical boundary is always lower than the one corresponding to the irrotational potential flow past a sphere (Moore 1963).

This paper is organized as follows. The set-up consisting of a uniform flow past a shear-free circular cylinder is described in $\S 2$. In $\S 3$ a perturbation expansion based asymptotic analysis is developed. The analysis includes formulation of parabolic boundary layer and wake vorticity transport equations in regions over which diffusion in the wall-normal or transverse directions overwhelms the diffusion along the streamwise direction. The flow in the neighbourhood of the rear stagnation region is quite distinct from the boundary layer and wake regions and lacks a dominant direction for diffusive or convective momentum transport. Our analysis of the flow in the rear stagnation region relies on a nonlinear, elliptic partial integro-differential equation that is formulated specifically to account for its distinct dynamics and non-local character. Our treatment of the flow in the vicinity of the rear stagnation is detailed in $\S 3.2$. In $\S 4$ an explicit expression for the drag coefficient is deduced from the composite flow field constructed by combining the solutions over the boundary layer, rear stagnation and wake regions. The principal results and key conclusions from this work are summarized in $\S 5$.

\section{The flow configuration}

We consider the uniform two-dimensional incompressible flow of a viscous Newtonian fluid past a stationary, perfectly slipping circular cylinder of diameter $D$ and infinite span. A schematic of the set-up is shown in figure 1. The set-up is conveniently described in a polar coordinate system $(r, \theta)$, with $r$ and $\theta$ denoting the radial and circumferential coordinates, respectively. We consider steady flow, the governing equations for which are 


\section{High-Reynolds-number flow past a shear-free cylinder}

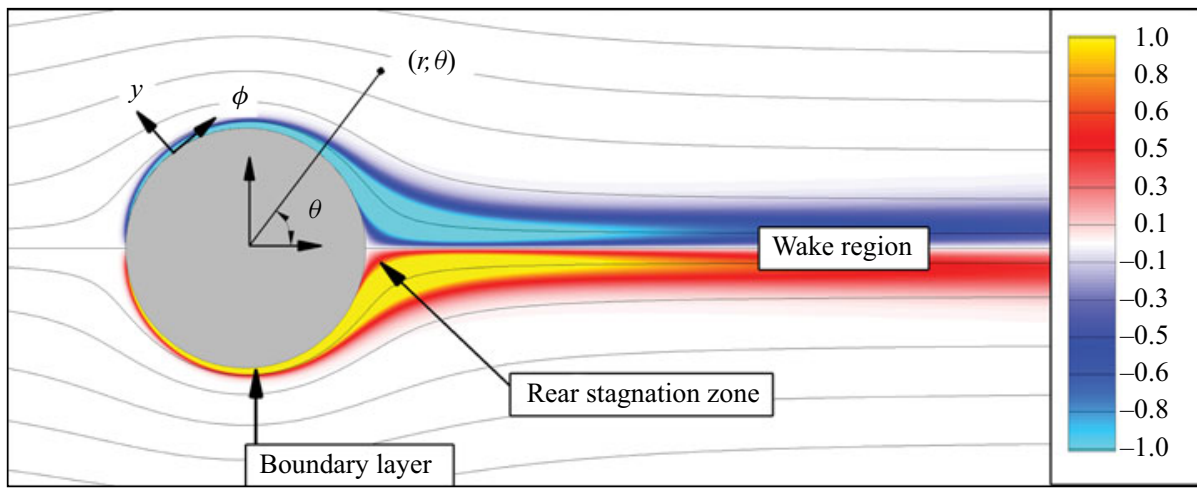

Figure 1. Schematic depicting the two-dimensional flow configuration consisting of a shear-free circular cylinder placed in a uniform crossflow of an incompressible Newtonian fluid. The coloured contours depict the vorticity field with the overlaid thin black lines representing the streamlines.

the stationary incompressible Navier-Stokes equations,

$$
\rho(\boldsymbol{u} \cdot \nabla) \boldsymbol{u}=-\nabla p+\mu \nabla^{2} \boldsymbol{u}, \quad \nabla \cdot \boldsymbol{u}=0,
$$

where $\boldsymbol{u}$ and $p$ denote the velocity and pressure fields, respectively, $\rho$ being the density and $\mu$, the dynamic viscosity of the fluid. The following no-through-flow and shear-free boundary conditions hold on the perfectly slipping cylinder's surface given by $r=a$ ( $a=$ $D / 2$ being the radius of the circular cylinder):

$$
\begin{gathered}
u_{r}(a, \theta)=0, \\
\tau_{r \theta}(a, \theta)=\left.0 \Leftrightarrow \frac{\partial u_{\theta}}{\partial r}\right|_{r=a}=\frac{u_{\theta}(a, \theta)}{a} .
\end{gathered}
$$

Here $u_{r}$ and $u_{\theta}$ denote the radial and circumferential components of the velocity vector $\boldsymbol{u}$. In $(2.2 b), \tau_{r \theta}$ represents the $r \theta$ component of the viscous stress tensor $\boldsymbol{\tau}=\mu(\nabla \boldsymbol{u}+$ $\left.(\nabla \boldsymbol{u})^{T}\right)$. Sufficiently far away from the cylinder, the flow attains a quiescent state corresponding to the uniform free stream along the positive $x$ direction. The far-field boundary conditions are given by

$$
u_{r} \rightarrow U_{\infty} \cos \theta, \quad u_{\theta} \rightarrow-U_{\infty} \sin \theta \quad \text { and } \quad p \rightarrow p_{\infty} \text { as } r \rightarrow \infty,
$$

where $U_{\infty} \boldsymbol{i}$ and $p_{\infty}$ denote the free-stream velocity and pressure, respectively, $\boldsymbol{i}$ being the unit vector in the $x$ direction. The flow is uniquely characterized by the Reynolds number $R e=\rho U_{\infty} D / \mu$.

\section{Asymptotic analysis}

Potential flow theory provides a simple means of determining an approximation to the flow field. For the present set-up, the irrotational potential flow given by

$\bar{u}_{r}=U_{\infty}\left(1-\frac{a^{2}}{r^{2}}\right) \cos \theta, \quad \bar{u}_{\theta}=-U_{\infty}\left(1+\frac{a^{2}}{r^{2}}\right) \sin \theta, \quad \bar{p}=p_{\infty}+\frac{1}{2} \rho\left(U_{\infty}^{2}-\bar{u}_{r}^{2}-\bar{u}_{\theta}^{2}\right)$,

is a solution of not just the incompressible Euler's equations but also the incompressible Navier-Stokes equations (2.1). The term viscous potential flow is often employed in 


\section{A. Kumar, N.M.A. Rehman, P. Giri and R.K. Shukla}

recognition of this important characteristic of the irrotational potential flow (Joseph, Funada \& Wang 2007).

The potential flow solution satisfies the far-field conditions $(2.3 a-c)$ and the no-through-flow condition $(2.2 a)$. The corresponding tangential shear stress on the cylinder's surface is given by $\bar{\tau}_{r \theta}(a, \theta)=\left(4 \mu U_{\infty} / a\right) \sin \theta$. Clearly, at any finite $R e$, the tangential shear stress over the cylinder surface predicted from the viscous potential flow solution does not vanish identically. The incompatibility between the potential flow solution and the shear-free boundary condition can also be viewed in terms of vorticity production at the shear-free interface. At the shear-free cylinder surface, a direct correspondence exists between the surface vorticity and the tangential surface velocity $\omega(a, \theta)=2 u_{\theta}(a, \theta) / a$, where $\omega$ denotes the vorticity component in the $z$ direction. Since $\bar{u}_{\theta}(a, \theta)$ is finite, the foregoing relationship would imply a contradictory existence of finite surface vorticity in an otherwise irrotational and inviscid flow field.

The apparent contradiction described above can be resolved by accounting for the existence of a boundary layer in the immediate vicinity of the shear-free cylinder surface. At high $R e$, this boundary layer, like its no-slip counterpart, is thin but significantly weaker as the relative change in the tangential surface velocity across it is $O\left(U_{\infty} R e^{-1 / 2}\right)$ (as opposed to $O\left(U_{\infty}\right)$ for a no-slip boundary). The vorticity produced in this thin boundary layer region is convected downstream first through a rear stagnation region and then eventually into a narrow wake. Modifications to the potential flow solution in each of these regions containing significant vorticity is necessary for elimination of an erroneous fore-aft symmetry in the irrotational flow field given by $(3.1 a-c)$. We note here that D'Alembert's paradox and the erroneous prediction of a zero-net-momentum-deficit wake are both direct consequences of the fore-aft symmetry of the potential flow solution. In the forthcoming subsections we develop interdependent asymptotic analyses necessary to account for the finite vorticity production, and its advection and diffusion into each of the distinct subregions (boundary layer, stagnation zone and wake) of the flow field.

\subsection{Boundary layer analysis}

At sufficiently high Reynolds numbers $(\operatorname{Re} \gg 1)$, the corrections to the potential flow solution that are necessary to enforce the shear-free condition $(2.2 b)$ can be sought within the broad purview of boundary layer theory. To analyse the flow characteristics in the boundary layer region, we define a scaled normal coordinate $y=(r-a) / a$ that is attached to the cylinder surface, along with a new tangential coordinate $\phi=\pi-\theta$. The origin of the $(y, \phi)$ coordinate system coincides with the forward stagnation point. In this newly defined $(y, \phi)$ coordinate system, we next express the flow variables as a superposition of the potential flow solution and a correction over it, i.e.

$$
u_{y}=\bar{u}_{y}+\tilde{u}_{y}, \quad u_{\phi}=\bar{u}_{\phi}+\tilde{u}_{\phi}, \quad p=\bar{p}+\tilde{p},
$$

where $\bar{u}_{y}$ and $\bar{u}_{\phi}$ are the normal and tangential potential flow velocity components in the $(y, \phi)$ coordinate system while $\bar{p}$ denotes the pressure deduced from the potential flow theory. The corresponding corrections to the velocity components and the pressure field are denoted by $\tilde{u}_{y}, \tilde{u}_{\phi}$ and $\tilde{p}$, respectively. Using (3.2b) and (3.1b) in $(2.2 b)$, we arrive at the following expression for the shear-free condition at the surface of the cylinder $y=0$,

$$
\left.\frac{\partial \tilde{u}_{\phi}}{\partial y}\right|_{y=0}=4 U_{\infty} \sin \phi+\left.\tilde{u}_{\phi}\right|_{y=0} .
$$

Inside the boundary layer region, the thickness of the boundary layer serves as an appropriate length scale along the normal direction. Denoting the boundary layer thickness 


\section{High-Reynolds-number flow past a shear-free cylinder}

scaled with $a$ by $\delta$, we find that within the boundary layer $(\partial / \partial y) \sim 1 / \delta$. The potential flow velocity components $\bar{u}_{\phi}$ and $\bar{u}_{y}$ in the boundary layer scale as $U_{\infty}$ and $U_{\infty} \delta$, respectively. Using the above facts and (3.3), we deduce that in the boundary layer $\tilde{u}_{\phi} \sim$ $U_{\infty} \delta$. The divergence-free constraint on the velocity field yields $\tilde{u}_{y} \sim U_{\infty} \delta^{2}$. To arrive at the boundary layer equations, we define a stretched coordinate system $\left(y^{*}, \phi\right)$ with a normalization in the normal direction: $y^{*}=y / \delta$. Furthermore, we define the following non-dimensional velocity components

$$
u_{\phi}^{*}=\frac{u_{\phi}}{U_{\infty}}, \quad u_{y}^{*}=\frac{u_{y}}{U_{\infty} \delta} .
$$

In the stretched coordinate system $\left(y^{*}, \phi\right)$, the circumferential momentum equation assumes the following form:

$$
\begin{aligned}
& u_{y}^{*} \frac{\partial u_{\phi}^{*}}{\partial y^{*}}+\frac{u_{\phi}^{*}}{\left(y^{*} \delta+1\right)} \frac{\partial u_{\phi}^{*}}{\partial \phi}+\frac{\delta u_{y}^{*} u_{\phi}^{*}}{\left(y^{*} \delta+1\right)}=-\frac{1}{\left(y^{*} \delta+1\right) \rho U_{\infty}^{2}} \frac{\partial p}{\partial \phi}+\frac{\mu}{\rho U_{\infty} a \delta^{2}} \\
& \quad \times\left(\frac{\partial^{2} u_{\phi}^{*}}{\partial y^{* 2}}+\frac{\delta}{\left(y^{*} \delta+1\right)} \frac{\partial u_{\phi}^{*}}{\partial y^{*}}-\frac{\delta^{2} u_{\phi}^{*}}{\left(y^{*} \delta+1\right)^{2}}+\frac{\delta^{2}}{\left(y^{*} \delta+1\right)^{2}} \frac{\partial^{2} u_{\phi}^{*}}{\partial \phi^{2}}+\frac{2 \delta^{3}}{\left(y^{*} \delta+1\right)^{2}} \frac{\partial u_{y}^{*}}{\partial \phi}\right) .
\end{aligned}
$$

The convective and the diffusive terms are both dominant in the boundary layer and, hence, the quantity $\mu /\left(\rho U_{\infty} a \delta^{2}\right)=2 /\left(\operatorname{Re} \delta^{2}\right)$ should be $O(1)$. Without loss of generality, we set $\delta=\sqrt{2 / R e}$.

In the boundary layer coordinates $\left(y^{*}, \phi\right)$, the radial momentum equation assumes the following non-dimensional form:

$$
\begin{aligned}
& \delta^{2} u_{y}^{*} \frac{\partial u_{y}^{*}}{\partial y^{*}}+\frac{\delta^{2} u_{\phi}^{*}}{\left(y^{*} \delta+1\right)} \frac{\partial u_{y}^{*}}{\partial \phi}-\frac{\delta u_{\phi}^{* 2}}{\frac{\left(y^{*} \delta+1\right)}{\rho}}=-\frac{1}{\rho U_{\infty}^{2}} \frac{\partial p}{\partial y^{*}} \\
& +\delta^{2}\left(\frac{\partial^{2} u_{y}^{*}}{\partial y^{* 2}}+\frac{\delta}{\left(y^{*} \delta+1\right)} \frac{\partial u_{y}^{*}}{\partial y^{*}}-\frac{\delta^{2} u_{y}^{*}}{\left(y^{*} \delta+1\right)^{2}}+\frac{\delta^{2}}{\left(y^{*} \delta+1\right)^{2}} \frac{\partial^{2} u_{y}^{*}}{\partial \phi^{2}}-\frac{2 \delta}{\left(y^{*} \delta+1\right)^{2}} \frac{\partial u_{\phi}^{*}}{\partial \phi}\right) .
\end{aligned}
$$

From the above relationship, one can deduce that in the boundary layer region, the contribution to the pressure from the inviscid potential flow $\bar{p}$ provides the centripetal force necessary for the flow to turn around the cylinder (signified through the underlined terms in the foregoing relationship (3.6)). The pressure perturbation $\tilde{p}$ therefore scales as $O\left(\delta^{2}\right)$.

Next, we invoke boundary layer approximations and retain only the most significant terms. Using (3.2a-c) in (3.5) along with the potential flow variables (3.1a-c), to a leading order, we obtain the following for the non-dimensional correction velocity component along the circumferential direction $\tilde{u}_{\phi}^{*}=\tilde{u}_{\phi} /\left(U_{\infty} \delta\right)$ :

$$
\frac{\partial^{2} \tilde{u}_{\phi}^{*}}{\partial y^{* 2}}=-2 y^{*} \cos \phi \frac{\partial \tilde{u}_{\phi}^{*}}{\partial y^{*}}+2 \sin \phi \frac{\partial \tilde{u}_{\phi}^{*}}{\partial \phi}+2 \tilde{u}_{\phi}^{*} \cos \phi .
$$

With only the leading-order terms retained, the boundary conditions $(2.2 b)$ and $(2.3 a-c)$ reduce to

$$
\tilde{u}_{\phi}^{*}=0 \text { at } \phi=0, \quad \tilde{u}_{\phi}^{*} \rightarrow 0 \quad \text { as } y^{*} \rightarrow \infty, \quad \frac{\partial \tilde{u}_{\phi}^{*}}{\partial y^{*}}=4 \sin \phi \text { at } y^{*}=0 .
$$

Note that the condition (3.8a) follows from the symmetry of the flow. 


\section{A. Kumar, N.M.A. Rehman, P. Giri and R.K. Shukla}

Boundary layer equations do not posses a characteristic scale and admit a dimension reduction through a similarity transformation. In typical planar and axisymmetric flows, the reduction in dimension facilitates simplification of the original partial differential equation and the associated boundary conditions into an ordinary differential equation and corresponding boundary conditions. A similarity transformation was also employed by Moore (1963) in his analysis of an axisymmetric boundary layer formed over a shear-free sphere. Given its wide-ranging success in a variety of flows lacking an inherent characteristic scale, it is natural to seek a similarity solution of the boundary layer equation for our present cylindrical set-up.

An analysis of the boundary layer equation (3.7) allows us to establish that a dimensional reduction of (3.7) is indeed possible, albeit for a slightly modified form of boundary condition (3.8c). Specifically, we show existence of a family of similarity solutions

$$
\tilde{u}_{\phi, n}^{*}=-\frac{\left(\sin \frac{\phi}{2}\right)^{2 n+2}}{\sqrt{\pi} \sin \phi} \int_{0}^{\pi} \exp \left(\frac{-\eta^{2}}{2 \cos ^{2}\left(\frac{\alpha}{2}\right)}\right) \sin ^{2 n+2}\left(\frac{\alpha}{2}\right) \mathrm{d} \alpha
$$

where $\eta=\sqrt{2} y^{*} \cos (\phi / 2)$ and $n=0,1,2, \ldots$ is a non-negative integer (see Appendix A for details). Each $\tilde{u}_{\phi, n}^{*}$ satisfies $(3.7),(3.8 a, b)$ and the boundary condition

$$
\frac{\partial \tilde{u}_{\phi, n}^{*}}{\partial y^{*}}=f_{n}(\phi)=\left[\sin \left(\frac{\phi}{2}\right)\right]^{2 n+1} \text { at } y^{*}=0 .
$$

Unfortunately, none of these unique solutions $\tilde{u}_{\phi, n}^{*}$ can individually be made to satisfy the boundary condition $(3.8 c)$. This is in striking contrast to the case of flow past a shear-free sphere wherein a single similarity transformation can be used to reduce the parabolic boundary layer equation and determine its closed-form solution (Moore 1963; Leal 2007).

The hindrance posed by the incompatibility between the derived similarity solutions and the boundary condition $(3.8 c)$ can be overcome by exploiting the linearity of the governing equation (3.7). Next, as shown in Appendix A, using the relationship

$$
4 \sin \phi=\sum_{n=0}^{\infty} w_{n}\left(\sin \frac{\phi}{2}\right)^{2 n+1}, \quad \text { where } w_{0}=8 \text { and } w_{n}=8 \prod_{i=1}^{n} \frac{(2 i-3)}{2 i} \text { for } n>0,
$$

the final solution to (3.7) and $(3.8 a-c)$ can be expressed as an infinite superposition of self-similar terms $\left(\tilde{u}_{\phi}^{*}=\sum_{n=0}^{\infty} w_{n} \tilde{u}_{\phi, n}^{*}\right)$ as follows:

$$
\tilde{u}_{\phi}^{*}=\frac{-4}{\sqrt{\pi}} \tan \left(\frac{\phi}{2}\right) \int_{0}^{\pi} \exp \left(\frac{-\eta^{2}}{2 \cos ^{2}\left(\frac{\alpha}{2}\right)}\right) \sin ^{2}\left(\frac{\alpha}{2}\right) \sqrt{1-\sin ^{2}\left(\frac{\alpha}{2}\right) \sin ^{2}\left(\frac{\phi}{2}\right)} \mathrm{d} \alpha .
$$

The above solution is in the form of superposition of infinitely many self-similar terms as opposed to the more commonly encountered solution consisting of a single self-similar term (as in the case of a shear-free sphere Moore 1963).

Vorticity produced at the shear-free cylinder's surface is convected and diffused throughout the boundary layer region. An estimate of this vorticity $\omega_{b l}$ can be obtained 
from the solution (3.12) as follows:

$$
\begin{aligned}
\omega_{b l}^{*} & =\frac{\omega_{b l}}{\left(U_{\infty} / a\right)}=\frac{a}{U_{\infty}}\left(\frac{\partial u_{\theta}}{\partial r}+\frac{u_{\theta}}{r}-\frac{1}{r} \frac{\partial u_{r}}{\partial \theta}\right)=-\frac{\partial \tilde{u}_{\phi}^{*}}{\partial y^{*}}+O(\delta) \\
& \approx-\sqrt{\frac{32}{\pi}} \sin \left(\frac{\phi}{2}\right) \int_{0}^{\pi} \eta \exp \left(\frac{-\eta^{2}}{2 \cos ^{2}\left(\frac{\alpha}{2}\right)}\right) \tan ^{2}\left(\frac{\alpha}{2}\right) \sqrt{1-\sin ^{2}\left(\frac{\alpha}{2}\right) \sin ^{2}\left(\frac{\phi}{2}\right)} \mathrm{d} \alpha .
\end{aligned}
$$

Here the superscript ' $*$ ' is used to denote non-dimensional vorticity.

\subsection{Rear stagnation region analysis}

The boundary layer analysis (BLA) of the previous subsection was based on the assumption of wall-normal gradients being significantly larger than the streamwise gradients. The surface-normal flow gradients diminish progressively with a gradual increase in the thickness of the boundary layer along the periphery of the cylinder. In the vicinity of the rear stagnation point $((y, \phi)=(0, \pi))$, as the flow undergoes a sharp turn, the reduction in flow gradients in the surface-normal direction is so significant that they become comparable to those in the circumferential direction. Thus, in the neighbourhood of the rear stagnation point, the boundary layer assumptions do not hold and, hence, the analysis of the previous section becomes unreliable. To complete the description of the flow over the entire shear-free cylinder boundary, we next analyse the flow in the vicinity of the rear stagnation region without invoking boundary layer assumptions.

The flow in the neighbourhood of the rear stagnation region involves a viscous boundary layer that effuses out into an inviscid flow (see figure 1 and the forthcoming analysis). This specific flow scenario corresponds closely to the one concerned with flow near a stagnation point on a rigid boundary (Harper 1963). In particular, close to the rear stagnation point we expect dominance of the convective terms over the diffusive terms and an appreciable variation in vorticity but not the velocity. To analyse the flow in the stagnation region, we adopt the $(y, \theta)$ coordinates ( $y$ as defined in $\S 3.1)$ so that the origin of the coordinate system coincides with the rear stagnation point. Our analysis closely follows the work of Harper (1963), in particular, (18) on page 148 of this cited work. Specifically, a scaling analysis of the most significant terms allows identification of two distinct regions with contrasting scenarios. The first region in which the convective terms dominate the viscous terms is centred around the rear stagnation point and extends to a distance of $O(1)$ into the boundary layer. The boundary layer assumptions themselves however are valid only beyond a certain distance from the rear stagnation point. This distance scales with the Reynolds number as $O\left(R e^{-1 / 4}\right)$. Therefore, there exists a region of dimension $O\left(R e^{-1 / 4}\right) \ll \theta \ll$ $O(1)$ over which the boundary layer assumptions are valid and the flow itself is inviscid.

We next develop a theoretical model for analysis of flow in the neighbourhood of the rear stagnation region. As shown below, the analysis independently provides justification for the aforementioned scaling arguments. In the vicinity of the stagnation region, i.e. towards the final stage of the boundary layer, vorticity is given by

$$
\lim _{\phi \rightarrow \pi} \omega_{b l}^{*}=-\sqrt{\frac{32}{\pi}} \int_{0}^{\pi} \eta \exp \left(\frac{-\eta^{2}}{2 \cos ^{2}\left(\frac{\alpha}{2}\right)}\right) \frac{\sin ^{2}\left(\frac{\alpha}{2}\right)}{\cos \left(\frac{\alpha}{2}\right)} \mathrm{d} \alpha, \quad \text { where } \eta=\frac{y \theta}{\sqrt{2} \delta} .
$$




\section{A. Kumar, N.M.A. Rehman, P. Giri and R.K. Shukla}

In this final stage of the boundary layer, the velocity variations are small. Consequently, in an overlap region within which the solutions from the BLA and the stagnation region analysis are both valid and should match, the distortion in the streamfunction $\tilde{\psi}$ is negligible compared with the contribution to the streamfunction from the potential flow $\bar{\psi}$. This region is off-axis and is located away from the singular rear stagnation point at $\theta=0$. In this region, the net streamfunction $\psi$ which is the sum of the above two, assumes the asymptotic form $\psi=\bar{\psi}+\tilde{\psi} \approx \bar{\psi} \approx 2 U_{\infty} a y \theta\left(\bar{\psi}=U_{\infty} a y(2+y) \sin \theta /(1+y)=\right.$ $2 U_{\infty}$ ay $\theta$ for $y \ll 1$ and $\left.\theta \ll 1\right)$. Using this asymptotic form, $\eta$ can be rewritten as

$$
\eta=\frac{\psi}{2 \sqrt{2} U_{\infty} a \delta}=\frac{\psi_{s}^{*}}{2 \sqrt{2}},
$$

where $\psi_{s}^{*}=\psi /\left(U_{\infty} a \delta\right)$ is the non-dimensional streamfunction near the rear stagnation point. Combining expressions (3.15) and (3.14) we obtain an expression for vorticity in the final stage of the boundary layer. Since the rear stagnation region is governed by inviscid dynamics, vorticity remains constant along streamlines. We therefore arrive at the following expression for vorticity in the stagnation region:

$$
\omega_{s}^{*}=-\frac{2}{\sqrt{\pi}} \int_{0}^{\pi} \psi_{s}^{*} \exp \left(\frac{-\psi_{s}^{* 2}}{16 \cos ^{2}\left(\frac{\alpha}{2}\right)}\right) \frac{\sin ^{2}\left(\frac{\alpha}{2}\right)}{\cos \left(\frac{\alpha}{2}\right)} \mathrm{d} \alpha .
$$

Here $\omega_{s}^{*}$ is the non-dimensional vorticity in the stagnation region $\left(\omega_{s}^{*}=\omega_{s} a / U_{\infty}\right)$.

The resultant vorticity from the end of the boundary layer region is simply transported along the streamlines into the rear stagnation region. In the overlap region the distortion in the streamfunction $\tilde{\psi}=O\left(R e^{-1}\right)$ is insignificant since the potential flow streamfunction $\bar{\psi}=O\left(R e^{-1 / 2}\right)$. The potential flow streamfunction $\bar{\psi}$ remains $O\left(R e^{-1 / 2}\right)$ in the stagnation region. Next, consider a potential flow streamline that is located $O(\delta)$ distance away from the cylinder's surface in the boundary layer region. In the rear stagnation region this streamline moves to $O\left(\delta^{1 / 2}\right)$ distance from the cylinder's surface and maintains the $O\left(\delta^{1 / 2}\right)$ distance from the line of symmetry near the stagnation point. The flow at the rear stagnation point corresponds closely to the flow in a right-angled corner. For such a stagnation point flow, both the directions $y$ and $\theta$ must be equally important or, in other words, $y \sim \theta$. Furthermore, the flow itself must turn around the corner so that $\psi \sim y \theta$. Next, noting that vorticity is produced solely from the distortion in the streamfunction, we deduce that $\tilde{\psi} \sim O\left(R e^{-1 / 2}\right)$ in the rear stagnation region. Moreover, $y, \theta \sim \delta^{1 / 2}$ or, equivalently, the size of the stagnation region is $O\left(R e^{-1 / 4}\right)$, in accordance with our assertion in the preceding paragraphs.

In view of the above arguments, to analyse the rear stagnation point flow, we next introduce an appropriately rescaled coordinate system $\left(y_{s}^{*}, \theta_{s}^{*}\right)$, where $y_{s}^{*}=y / \delta^{1 / 2}$ and $\theta_{s}^{*}=\theta / \delta^{1 / 2}$. In our present planar set-up, an absence of vortex stretching mechanisms suggests that the vorticity level in the stagnation zone matches that in the boundary layer region. Thus, $\omega_{s}^{*} \sim O(1)$, which can be inferred from (3.16) as well. For a non-dimensional vorticity that scales as $O(1)$, the associated non-dimensional velocity corrections to the inviscid flow must necessarily scale as the characteristic non-dimensional length scale, or equivalently $O\left(R e^{-1 / 4}\right)$.

An inspection of the expression $(3.1 a, b)$ reveals that in the rear stagnation region, the potential velocity components themselves scale as $O\left(R e^{-1 / 4}\right)$. This would imply that both the correction and the potential flow components exhibit similar scalings and are therefore 


\section{High-Reynolds-number flow past a shear-free cylinder}

comparable in magnitude. Expressing vorticity as the curl of the velocity vector and retaining only the significant terms as determined from the appropriate scales in the rear stagnation region, we deduce that

$$
\frac{\partial \tilde{u}_{\theta_{(s)}}^{*}}{\partial y_{s}^{*}}-\frac{\partial \tilde{u}_{y_{(s)}^{*}}^{*}}{\partial \theta_{s}^{*}}=\omega_{s}^{*}
$$

where $\tilde{u}_{\theta_{(s)}}^{*}=\tilde{u}_{\theta} /\left(U_{\infty} \delta^{1 / 2}\right)$ and $\tilde{u}_{y_{(s)}}^{*}=\tilde{u}_{r} /\left(U_{\infty} \delta^{1 / 2}\right)$ are the non-dimensional correction velocity components in the circumferential and radial directions, respectively. The correction velocity components can be expressed in terms of the distortion to the streamfunction as follows:

$$
\tilde{u}_{y_{(s)}}^{*}=\frac{\partial \tilde{\psi}_{s}^{*}}{\partial \theta_{s}^{*}}, \quad \tilde{u}_{\theta_{(s)}}^{*}=-\frac{\partial \tilde{\psi}_{s}^{*}}{\partial y_{s}^{*}} .
$$

Using (3.16) and $(3.18 a, b)$ in the relationship (3.17), we obtain the following governing equation for the correction velocity induced distortion in the streamfunction:

$$
\frac{\partial^{2} \tilde{\psi}_{s}^{*}}{\partial y_{s}^{* 2}}+\frac{\partial^{2} \tilde{\psi}_{s}^{*}}{\partial \theta_{s}^{* 2}}=-\omega_{s}^{*}=\frac{2}{\sqrt{\pi}} \int_{0}^{\pi}\left(\tilde{\psi}_{s}^{*}+\bar{\psi}_{s}^{*}\right) \exp \left(\frac{-\left(\tilde{\psi}_{s}^{*}+\bar{\psi}_{s}^{*}\right)^{2}}{16 \cos ^{2}\left(\frac{\alpha}{2}\right)}\right) \frac{\sin ^{2}\left(\frac{\alpha}{2}\right)}{\cos \left(\frac{\alpha}{2}\right)} \mathrm{d} \alpha .
$$

Here $\tilde{\psi}_{s}^{*}=\tilde{\psi} /\left(U_{\infty} a \delta\right)$ and $\bar{\psi}_{s}^{*}=\bar{\psi} /\left(U_{\infty} a \delta\right)=2 y_{s}^{*} \theta_{s}^{*}$ are the non-dimensional distortion and potential flow streamfunctions in the rear stagnation region, respectively. The correction flow becomes unidirectional in the final stage of the boundary layer and towards the beginning of the wake. These facts combined with the no-through-flow condition at the cylinder surface and the symmetry condition along the rear axis of symmetry $(\theta=0)$, give rise to the following boundary conditions on the distortion streamfunction $\tilde{\psi}_{s}^{*}$ :

$$
\tilde{\psi}_{s}^{*}=0 \text { at } y_{s}^{*}, \theta_{s}^{*}=0, \quad \frac{\partial \tilde{\psi}_{s}^{*}}{\partial y_{s}^{*}} \rightarrow 0 \quad \text { as } y_{s}^{*} \rightarrow \infty, \quad \frac{\partial \tilde{\psi}_{s}^{*}}{\partial \theta_{s}^{*}} \rightarrow 0 \quad \text { as } \theta_{s}^{*} \rightarrow \infty .(3.20 a-c)
$$

We note here that owing to the relationship (3.16), the above homogeneous boundary condition on the distortion streamfunction enforces the shear-free condition as well (to a leading order, the homogeneous boundary condition for surface vorticity and the shear-free constraint at the cylinder's boundary are equivalent).

Equation (3.19) is an elliptic partial integro-differential equation that involves a non-local source term. The nonlinearity of (3.19) makes its analytical tractability extremely challenging, if not impossible. This complication arises principally because the perturbations to the potential flow turn out to be comparable to the flow itself in the rear stagnation region. A similar nonlinearity was encountered in the planar analysis of two-dimensional stagnation point flow (Harper 1963). We note here that in an axisymmetric configuration, a reduction in the magnitude of vorticity due to contraction of vortex lines in the stagnation region ensures that perturbations remain insignificant in comparison with the base potential flow (Harper \& Moore 1968). This insignificance of the perturbations facilitates full analytical resolution of the axisymmetric flow in the vicinity of the rear stagnation region of a shear-free spherical surface (Moore 1963).

To make further progress, we solve (3.19) subject to boundary conditions $(3.20 a-c)$ numerically using standard approximation techniques. Note that the vorticity field from the boundary layer solution (3.13) is regular and bounded $\left(\left|\omega_{b l}^{*}\right| \leq 4\right)$ and, hence, the 

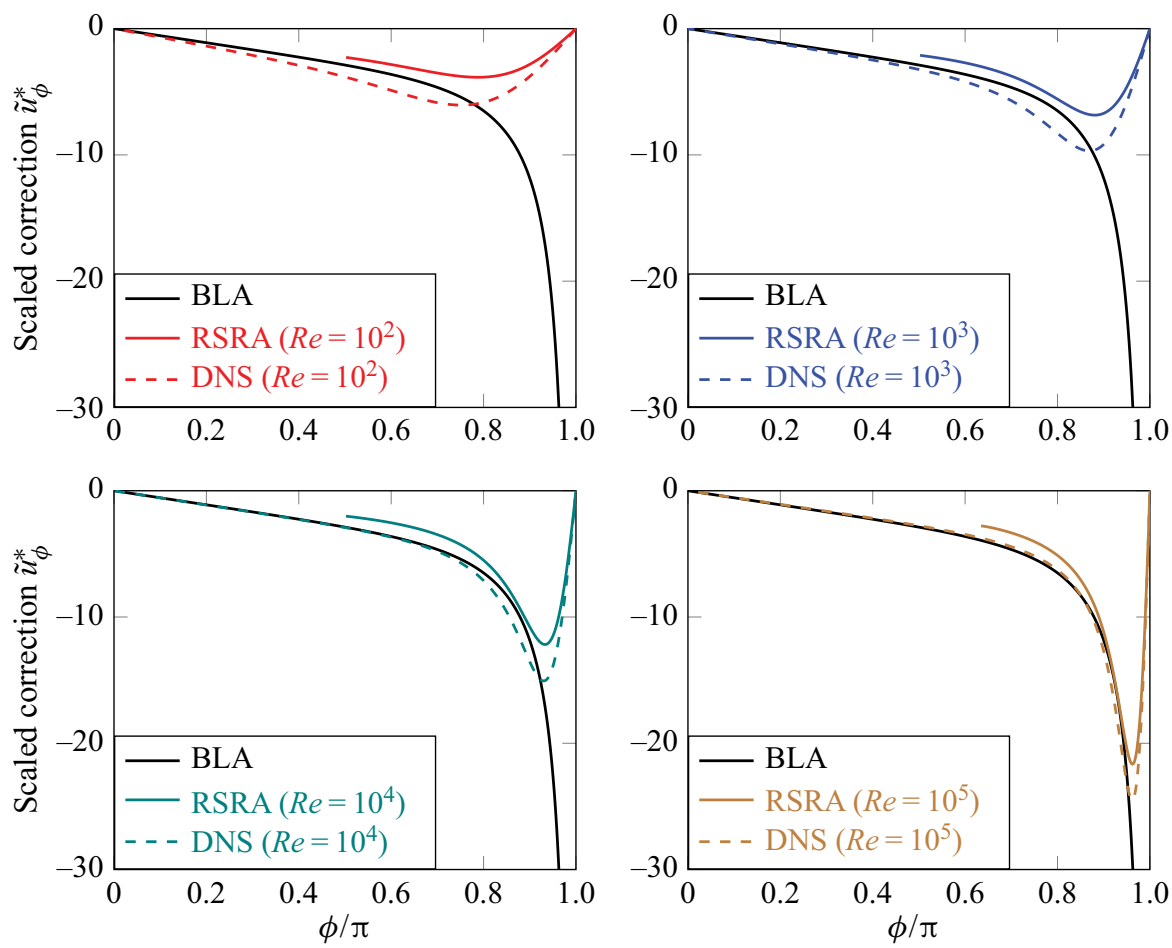

Figure 2. The scaled correction in the tangential surface velocity $\left(\tilde{u}_{\phi}^{*}=\sqrt{\operatorname{Re} / 2}\left(u_{\phi}-\bar{u}_{\phi}\right) / U_{\infty}\right)$ along the shear-free cylinder surface at Reynolds numbers $10^{2}, 10^{3}, 10^{4}$ and $10^{5}$. Predictions from the boundary layer analysis (BLA) and the rear stagnation region analysis (RSRA) are indicated using solid black and solid coloured lines, respectively. Dotted coloured lines represent results from direct numerical simulations (DNS).

right-hand side of (3.19) and the corresponding integral solution are both regular. This regularity enables discretization of (3.19) using standard approximation techniques. Our numerical implementation is detailed in Appendix B. Our analysis of the rear stagnation and the boundary layer regions provides a complete description of the flow along the periphery and in the immediate neighbourhood of the shear-free cylinder. Figure 2 depicts the scaled tangential velocity correction $\tilde{u}_{\phi}^{*}=\sqrt{R e / 2}\left(\tilde{u}_{\phi} / U_{\infty}\right)$ along the surface of the cylinder predicted from our analysis of the present and preceding subsections at $R e=10^{2}, 10^{3}, 10^{4}$ and $10^{5}$. Coloured solid lines in figure 2 illustrate the trends for the rear stagnation region obtained from the numerical solution of (3.19) and (3.20a-c). The numerical solution is only valid in the neighbourhood of the rear stagnation point. Hence, the coloured solid lines extend only over a limited range of $\phi$ representing a portion of the rearward cylinder surface. The scaled tangential velocity correction increases in magnitude and subsequently decreases after attaining a maxima as one traverses from the rear stagnation point to the forward stagnation point along the cylinder surface. This broad trend is observed at all the four Reynolds numbers, with both the magnitude of the peak and the spread in $\tilde{u}_{\phi}^{*}$ strongly dependent on the Reynolds number. We note here that this apparent dependence on $R e$ is entirely due to the disparity between normalization scales employed in figure 2 and the relevant characteristic scales in the stagnation region ( $\delta$ vs $\sqrt{\delta}$ for the velocity scale for instance).

Solid black lines in figure 2 depict the scaled tangential velocity correction deduced from the BLA (i.e. from (3.12)). Owing to the boundary layer specific characteristic 


\section{High-Reynolds-number flow past a shear-free cylinder}

scale based normalization employed in figure $2, \tilde{u}_{\phi}^{*}$ given by (3.12) is independent of the Reynolds number and so are the solid black lines depicted in each of the four frames of figure 2 . The scaled correction $\tilde{u}_{\phi}^{*}$ grows monotonically with $\phi$. The growth is particularly pronounced over the rearward cylinder surface $(\phi \geq \pi / 2)$. In particular, $\tilde{u}_{\phi}^{*}$ becomes unbounded in the vicinity of the rear stagnation point. This unphysical divergence of $\tilde{u}_{\phi}^{*}$ as $\phi \rightarrow \pi$ is a direct consequence of the breakdown of the assumptions inherent in the BLA.

To determine $\tilde{u}_{\phi}^{*}$ over the entire cylinder periphery, we must employ complementary boundary layer and stagnation region analyses over their respective domains of validity. Furthermore, a matching procedure must be invoked over the overlap region $O\left(\operatorname{Re}^{-1 / 4}\right) \ll$ $\theta \ll O(1)$ to obtain a uniformly valid solution $\tilde{u}_{\phi}^{*}$ over the entire range $0 \leq \phi \leq \pi$. The dependence of $\tilde{u}_{\phi}^{*}$ on $\phi$, as illustrated in figure 2 , suggests that such a matching is indeed possible, albeit at sufficiently high $R e$, which is when our asymptotic analyses of boundary layer and stagnation regions are strictly valid (i.e. in the limit $\operatorname{Re} \rightarrow \infty$ ).

Our theoretical results can be directly compared with the predictions from detailed simulations. To this end, we perform direct numerical simulations (DNS) of a two-dimensional unsteady incompressible flow past a shear-free circular cylinder using a well-established technique in polar cylindrical coordinates (see Shukla \& Zhong (2005) and Shukla, Tatineni \& Zhong (2007) for details and verification tests). In brief, our simulation methodology relies on a combination of tenth-order compact finite difference and Fourier-spectral schemes (in $r$ and $\theta$ coordinates, respectively) and a second-order semi-implicit projection scheme (Hugues \& Randriamampianina 1998) for spatiotemporal discretization. In all our runs we employ spatiotemporal resolutions necessary to resolve the thin boundary layers and enforce the Courant-Friedrichs-Lewy convective stability criterion. We also perform long-time simulations on successively refined meshes to ensure mesh independence of the computed solution.

The predictions from our DNS runs are depicted in figure 2 alongside results from the asymptotic BLA and the rear stagnation region analysis (RSRA). The comparison is quite encouraging and more so at high Reynolds numbers. Specifically, over the windward section of the cylinder's boundary, discernible deviations between the DNS results and BLA are evidenced at $R e=10^{2}$. The deviations reduce progressively with an increase in the Reynolds number. In particular, at the highest $R e=10^{5}$, the predictions from BLA agree remarkably well with the DNS results all the way up to the location at which a maximum in the magnitude of $\tilde{u}_{\phi}^{*}$ is encountered. We observe trends similar to those noted for the BLA in the rear stagnation region as well. Appreciable deviations are evidenced between the predictions from RSRA and the DNS results at Reynolds numbers of $10^{2}$ and $10^{3}$. The deviations reduce considerably at $R e=10^{4}$ and are only marginal at the highest Reynolds number of $R e=10^{5}$ investigated in our work. Overall, at a given $R e$, the discrepancy between DNS and RSRA is discernibly more pronounced than the discrepancy between DNS and BLA. This increased discrepancy is attributable to the more stringent restrictions on the Reynolds numbers that are necessary in RSRA $\left(\operatorname{Re}^{-1 / 2} \ll 1\right.$ for the BLA vs $R e^{-1 / 4} \ll 1$ in the case of RSRA).

We note here that viscous terms do become important in the immediate vicinity of the rear stagnation point. The presence of such a viscous sublayer region in general stagnation point flows was mentioned in Harper (1963). The scale of this relatively thin region is $O\left(R e^{-3 / 4}\right)$, a fact that can be readily established by appealing to the vorticity transport equation with consideration of viscous diffusion. The scale of this region implies that its contribution to the leading-order terms of interest in this work is insignificant. 


\section{A. Kumar, N.M.A. Rehman, P. Giri and R.K. Shukla}

\subsection{Analysis of the wake region}

To complete our description of the vortical regions of the flow field, we next develop an asymptotic analysis of the narrow downstream wake region into which the vorticity generated at the shear-free cylindrical surface is eventually convected (see figure 1). We adopt a Cartesian coordinate system $(x, z)$ where both the scaled coordinates $x$ and $z$ are normalized with respect to the cylinder's radius $a$. The origin of the coordinate system coincides with the rear stagnation point with the $x$-axis pointing along the streamwise direction.

As argued in the earlier subsection, an absence of a vortex stretching mechanism in the present planar configuration implies that the non-dimensional vorticity $\left(\omega^{*}=a \omega / U_{\infty}\right)$ scales as $O(1)$ in the boundary layer and stagnation regions, and crucially, in the initial stages of the wake region as well. The wake thickness is the appropriate length scale for characterization of the wake region. Denoting the wake thickness normalized with the cylinder radius by $\delta_{w}$ and making use of the non-dimensional vorticity scale of $O(1)$, we deduce that the correction velocity in the streamwise direction $\tilde{u}_{x}$ should scale as $O\left(\delta_{w}\right)$. Applying the divergence-free condition on the correction velocity field, we obtain $\tilde{u}_{z} \sim$ $O\left(\delta_{w}^{2}\right)$ as the characteristic scale for the component of the correction velocity in the $z$ direction.

In the wake region the inviscid base flow velocity components deduced from the potential flow theory can be shown to scale as $\bar{u}_{x} \sim O(1)$ and $\bar{u}_{z} \sim O\left(\delta_{w}\right)$, respectively. Balancing out the convective and the diffusive terms in the wake, in a way similar to our analysis of the boundary layer region, we obtain $\delta_{w} \sim \delta$. Without loss of generality, we set $\delta_{w}=\delta$. Using the characteristic scales described above, we next define appropriately normalized spatial coordinates and the base potential flow and correction velocity components as follows:

$$
z^{*}=\frac{z}{\delta}, \quad \bar{u}_{x}^{*}=\frac{\bar{u}_{x}}{U_{\infty}}, \quad \bar{u}_{z}^{*}=\frac{\bar{u}_{z}}{U_{\infty} \delta}, \quad \tilde{u}_{x}^{*}=\frac{\tilde{u}_{x}}{U_{\infty} \delta}, \quad \tilde{u}_{z}^{*}=\frac{\tilde{u}_{z}}{U_{\infty} \delta^{2}} .
$$

With the above definitions in place, the equation that governs the distribution of non-dimensional vorticity $\omega_{w}^{*}=\omega_{w} a / U_{\infty}$ in the wake region assumes the following linearized form:

$$
\bar{u}_{x_{0}}^{*} \frac{\partial \omega_{w}^{*}}{\partial x}+\bar{u}_{z_{0}}^{*} \frac{\partial \omega_{w}^{*}}{\partial z^{*}}=\frac{\partial^{2} \omega_{w}^{*}}{\partial z^{* 2}} .
$$

Here we have only retained the leading-order terms, and, $\bar{u}_{x_{0}}^{*}$ and $\bar{u}_{z_{0}}^{*}$ denote the zeroth-order terms in an expansion of $\bar{u}_{x}^{*}$ and $\bar{u}_{z}^{*}$ about $z^{*}=0$, respectively. Imposition of symmetry of the flow field about $z^{*}=0$ and the irrotationality of the far-field flow leads to the boundary conditions

$$
\omega_{w}^{*}=0 \text { at } z^{*}=0, \quad \omega_{w}^{*} \rightarrow 0 \text { as } z^{*} \rightarrow \infty .
$$

Moreover, we require the solution $\omega_{w}^{*}$ of (3.22) subject to boundary conditions $(3.23 a, b)$ to be such that it results in a drag-producing far wake with a constant momentum deficit. The general solution of $(3.22)$ and $(3.23 a, b)$ that satisfies the aforementioned criterion assumes the form

$$
\omega_{w}^{*}=\sum_{n} \frac{\lambda_{n} \zeta_{n} \exp \left(-\frac{\zeta_{n}^{2}}{2}\right)}{\left(x+1+\frac{1}{x+1}+c_{n}\right)}, \quad \text { where } \zeta_{n}=\frac{z^{*}\left(1-\frac{1}{(x+1)^{2}}\right)}{\sqrt{2}\left(x+1+\frac{1}{x+1}+c_{n}\right)^{1 / 2}} .
$$




\section{High-Reynolds-number flow past a shear-free cylinder}

Like the solution (3.12) of the boundary layer (3.7), the above solution is in the form of superposition of a family of infinite self-similar wake solutions each with strength $\lambda_{n}$ and centred around the coordinates $\left(-\left(c_{n}+1\right), 0\right)$. We require the yet to be determined constants $\lambda_{n}$ and $c_{n}$ in the above expression (3.24) to be such that the asymptotic forms of $\omega_{w}^{*}$ and $\omega_{b l}^{*}$ match in the rear stagnation region. Note that since the flow in the rear stagnation region is symmetric in $y$ and $\theta$, the vorticity in the beginning of the wake region must match the one associated with the final stage of the boundary layer. The transition of the flow from the stagnation region to the wake region is thus, to leading order, an inversion of the flow coming from the boundary layer into the stagnation region. Therefore, the distortion in the streamfunction becomes smaller at the right side of the stagnation region (beginning of the wake region). Consequently, the vorticity in the beginning of the wake region is of a form given by the expression (3.14). Matching the general solution of the vorticity in the wake region given by (3.24) and the vorticity distribution given by (3.14) allows us to determine the final form of $\omega_{w}^{*}$,

$$
\omega_{w}^{*}=-\sqrt{\frac{2}{\pi}} \int_{-2}^{2} \frac{\zeta_{\kappa} \exp \left(-\frac{\zeta_{\kappa}^{2}}{2}\right) \sqrt{4-\kappa^{2}}}{\left(x+1+\frac{1}{x+1}-\kappa\right)} \mathrm{d} \kappa, \quad \text { where } \zeta_{\kappa}=\frac{z^{*}\left(1-\frac{1}{(x+1)^{2}}\right)}{\sqrt{2}\left(x+1+\frac{1}{x+1}-\kappa\right)^{1 / 2}} .
$$

At a location far downstream, i.e. when $x \gg 1,(3.25)$ assumes the asymptotic form

$$
\omega_{w}^{*} \rightarrow-\frac{2 \sqrt{\pi} z^{*}}{x^{3 / 2}} \exp \left(-\frac{z^{* 2}}{4 x}\right) \quad \text { for } x \rightarrow \infty .
$$

The above asymptotic expression is precisely in the form of the well-known self-similar solution for the defect velocity in the laminar wake of a planar body with a given drag coefficient (Schlichting \& Gersten 2003). A comparison with the solution for the defect velocity from Schlichting \& Gersten (2003) reveals that sufficiently far away from the cylinder surface, the wake solution (3.25) exhibits an asymptotic decay rate that corresponds to a drag coefficient of $16 \pi / R e$. As shown in the forthcoming section, to a leading order, the drag coefficient associated with the flow past a shear-free circular cylinder matches the aforementioned value of $16 \pi / R e$ exactly. Thus, our combined asymptotic analysis of the boundary layer, rear stagnation and the wake regions is self-consistent in that the drag predicted from the analysis, as derived in the forthcoming section, equals the one deduced from the far-field wake signature.

\section{Viscous dissipation and drag coefficient}

The viscous corrections to the inviscid base state induce a streamwise asymmetry in the otherwise symmetric stress field. The viscous correction-induced circumferential asymmetry in the surface stress gives rise to a non-vanishing drag force. We next attempt to quantify the finite drag force and in the process resolve D'Alembert's paradox specifically for the configuration being investigated.

An expression for the net drag force can be derived by summing up the componentwise contributions from the pressure and viscous stresses along the $x$ direction. Given the asymptotic form of the solution, such an expression would be tantamount to summing up the contributions from the zeroth-order inviscid base flow and the first-order viscous correction to it. Owing to the symmetry of the inviscid base flow, the contributions to the drag from the zeroth-order terms in the asymptotic expansion for pressure and viscous 
stresses must necessarily sum up to zero, thus leading to D'Alembert's paradox. An expression for the first-order term in the asymptotic expansion of the drag can be readily determined from the solutions derived in the preceding section. Alternatively, to ensure consistency with the first-order correction term in the wake solution, we may intuitively assert that the first-order term in the asymptotic expansion of the drag coefficient must necessarily equal $16 \pi / R e$.

Since we have determined the correctional flow field only to a leading first order, the aforementioned direct evaluation method can not be used to determine any subsequent higher-order terms in the asymptotic expansion of the drag coefficient (Kang \& Leal 1988). Moreover, a direct evaluation of the drag coefficient relies explicitly on the pressure distribution over the surface of the cylinder. Determination of the first-order term in the asymptotic expansion of a pressure field is complicated by the inclusion of first-order effects in the surface vorticity.

Notwithstanding these complications, an improved estimate of the drag coefficient without any explicit involvement of surface pressure distribution can nonetheless be deduced by appealing to the overall mechanical energy balance in the flow. Specifically, in any generic drag-producing, thrust generating or self-propelling configuration with finite tangential surface motion, the net energy dissipation rate must match the sum of the rates at which work must be done to counteract the drag force and sustain the tangential boundary motion (Arakeri \& Shukla 2013). Thus, one may express the non-dimensional total viscous dissipation as a power loss coefficient, which for a shear-free cylinder with vanishing tangential surface stress must equal the drag coefficient (Shukla \& Arakeri 2013), i.e.

$$
C_{P L}=\frac{\mu}{\rho U_{\infty}^{3} a} \int_{\Omega} \bar{\Phi}_{v} \mathrm{~d} \Omega=C_{D}-\frac{1}{\rho U_{\infty}^{3}} \int_{0}^{2 \pi} \tau_{r \theta}(a, \theta) u_{\theta}(a, \theta) \mathrm{d} \theta=C_{D},
$$

where $\Phi_{v}=(\boldsymbol{\tau}: \nabla \boldsymbol{u}) / \mu$ is the dissipation function with $\Omega$ denoting the entire flow domain excluding the cylinder's interior. Furthermore, using $\boldsymbol{\tau}: \nabla \boldsymbol{u}=\mu\left(|\boldsymbol{\omega}|^{2}+\nabla \cdot(\boldsymbol{u} \cdot\right.$ $\nabla \boldsymbol{u})$ ) the drag coefficient can be conveniently expressed in terms of the total vorticity and the tangential surface velocity as (Lamb 1932; Shukla \& Arakeri 2013)

$$
C_{D}=C_{P L}=\frac{\mu}{\rho U_{\infty}^{3} a}\left(2 \int_{0}^{2 \pi} u_{\theta}^{2}(a, \theta) \mathrm{d} \theta+\int_{\Omega} \omega^{2} \mathrm{~d} \Omega\right) .
$$

A direct substitution of the irrotational potential flow solution $\left(\bar{u}_{\theta}(a, \theta)=-2 U_{\infty} \sin \theta\right.$ and $\bar{\omega}=0$ ) into the relationship (4.2) yields $\bar{C}_{P L}=16 \pi / R e$ for the power loss coefficient (Shukla \& Arakeri 2013). Thus, the power loss coefficient deduced from the zeroth-order term in the asymptotic expansion of the flow solution precisely equals the drag coefficient estimated by retaining the zeroth and first-order terms in the asymptotic expansion of the flow solution. Hence,

$$
C_{D}^{(1)}=\bar{C}_{P L}=\frac{16 \pi}{R e},
$$

where the superscript '(1)' signifies the order to which the terms in the asymptotic expansion of the drag coefficient are retained. Note that in the case of a shear-free sphere, a similar observation leads to Levich's celebrated result $C_{D}^{(1)}=48 / \operatorname{Re}$ for drag on a spherical bubble (Levich 1949). In essence, each term in the asymptotic expansion of the power loss coefficient matches a subsequent higher-order term in the asymptotic expansion of the drag coefficient. Hence, an improved estimate for the drag coefficient can simply be determined through a direct substitution of the potential flow solution and the first-order correctional terms in the relationship (4.2). 


\section{High-Reynolds-number flow past a shear-free cylinder}

We begin by estimating the contribution from the tangential surface velocity (first term that appears in the parenthesis on the right-hand side of (4.2)). To a leading order,

$$
2 \int_{0}^{2 \pi} u_{\theta}^{2}(a, \theta) \mathrm{d} \theta \approx 2 \int_{0}^{2 \pi} \bar{u}_{\theta}^{2}(a, \theta) \mathrm{d} \theta+4 \int_{0}^{2 \pi} \bar{u}_{\theta}(a, \theta) \tilde{u}_{\theta}(a, \theta) \mathrm{d} \theta .
$$

The first term on the right-hand side of the above expression (4.4) corresponds to the inviscid potential flow and, therefore, follows from the analysis in the preceding paragraph. The integrand in the second term on the right-hand side of (4.4) is non-singular. A numerical evaluation of the second term on the right-hand side of (4.4) yields

$$
\frac{2 \mu}{\rho U_{\infty}^{3} a} \int_{0}^{2 \pi} u_{\theta}^{2}(a, \theta) \mathrm{d} \theta \approx \frac{16 \pi}{R e}\left(1-\frac{6.20}{\sqrt{R e}}\right) .
$$

Next we consider the contribution from the total vorticity. Owing to the symmetry of the flow, the integral term on the extreme right of (4.2) can be recast as

$$
\frac{\mu}{\rho U_{\infty}^{3} a} \int_{\Omega} \omega^{2} \mathrm{~d} \Omega=\frac{2 \mu}{\rho U_{\infty}^{3} a} \int_{\Omega_{u}} \omega^{2} \mathrm{~d} \Omega
$$

where $\Omega_{u}$, with the subscript ' $u$ ' representing upper, denotes the region $(r, \theta): 0 \leq \theta \leq$ $\pi, a \leq r<\infty$. Substitution of a uniformly valid vorticity field over the entire domain $\Omega_{u}$ would yield an estimate for the integral (4.6). For the present set-up, such an evaluation of (4.6) would amount to summing up individual contributions from the vorticity distributions over the boundary layer, rear stagnation and wake regions. The contributions from the boundary layer and the wake regions can be deduced through a direct substitution of the respective vorticity distributions (3.13) and (3.25) into the integrand of (4.6). As detailed in $\$ 3.2$, owing to the nonlinearity of (3.19), the vorticity distribution in the stagnation region is determined numerically and, therefore, the contribution to (4.6) from the rear stagnation region must be computed via numerical quadrature.

A direct quadrature-based numerical computation of the contribution to (4.6) from the rear stagnation region is however problematic due to a logarithmic singularity in $\psi_{s}$. Specifically, the singularity in $\omega_{s}$ leads to a numerical prediction that diverges with mesh refinement. The singularity is generic in that it exists in planar stagnation point flows (Harper 1963) and its full resolution for the present specific configuration would presumably require accounting for the presence of a viscous layer adjacent to the shear-free cylindrical surface. For an estimation of the contribution to (4.6) from $\omega_{s}$, it suffices to address the aforementioned lack of convergence through a singularity subtraction technique that regularizes the integrand.

To regularize the integrand in (4.6) in the rear stagnation region, we devise a desingularization technique wherein we split (4.6) as follows:

$$
\frac{2 \mu}{\rho U_{\infty}^{3} a} \int_{\Omega_{u}} \omega^{2} \mathrm{~d} \Omega=\underbrace{\frac{2 \mu}{\rho U_{\infty}^{3} a} \int_{\Omega_{u}} \omega_{b l, w}^{2} \mathrm{~d} \Omega}_{\mathrm{I}}+\underbrace{\frac{2 \mu}{\rho U_{\infty}^{3} a} \int_{\Omega_{s}}\left(\omega_{s}^{2}-\lim _{\theta \rightarrow 0} \omega_{b l}^{2}\right) \mathrm{d} \Omega}_{\mathrm{II}} .
$$

Here $\Omega_{s}$ denotes the upper half of the stagnation region. Our approach of constructing uniformly valid asymptotic expansions ensures that in the vicinity of the rear stagnation point, the form of singularity in the boundary layer and wake vorticity distributions matches that of the rear stagnation region vorticity distribution. Our desingularization technique exploits this similarity in the nature of the singularity to effectively eliminate 


\section{A. Kumar, N.M.A. Rehman, P. Giri and R.K. Shukla}

it. Specifically, the integrand of (II) is free of the singularity and, therefore, amenable to accurate numerical evaluation using standard quadrature rules. The first integral (I) in the above expression (4.7) is evaluated over the entire upper domain by making use of the analytical distributions (3.13) and (3.25) for vorticity in the boundary layer and the wake regions, respectively. Furthermore, in evaluation of (I) over the portion of $\Omega_{u}$ that coincides with the rear stagnation region, we employ extensions of the boundary layer/wake region vorticity $\left(\omega_{b l}\right.$ or $\left.\omega_{w l}\right)$ into the rear stagnation region and not the actual vorticity in the rear stagnation region $\left(\omega_{s}\right)$ itself.

The first integral (I) involves two regions, each with distinct solutions; one from the boundary layer region given by (3.13) and the other given by (3.25) associated with the wake region. We therefore rearrange (4.6) as follows:

$$
\frac{2 \mu}{\rho U_{\infty}^{3} a} \int_{\Omega_{u}} \omega_{b l, w}^{2} \mathrm{~d} \Omega=\frac{2 \mu}{\rho U_{\infty}^{3} a} \int_{\Omega_{b l}} \omega_{b l}^{2} \mathrm{~d} \Omega+\frac{2 \mu}{\rho U_{\infty}^{3} a} \int_{\Omega_{w}} \omega_{w}^{2} \mathrm{~d} \Omega .
$$

To make further progress a partitioning of the domain $\Omega_{u}$ into the boundary layer and wake regions $\Omega_{b l}$ and $\Omega_{w}$ is necessary. Since the solutions in the boundary layer and wake regions assume similar asymptotic forms in the rear stagnation region, it can be reasonably asserted that the curve $C$ dividing the two regions passes through the rear stagnation point. Importantly, as established below, so long as the dividing curve $C$ originates from the rear stagnation point, the leading-order terms in (I) (that we are interested in) remain insensitive to the specifics of $C$.

In the asymptotic limit $R e \rightarrow \infty$, the vorticity field undergoes a sharp decay away from the relatively thin boundary layer, rear stagnation and wake regions. The integrands in (4.8) are therefore finite only over these extremely thin regions with finite vorticity. We therefore expect the dependence of (4.8) on the specifics of the dividing curve $C$ to remain localized to the immediate neighbourhood of the rear stagnation point. Consequently, we may limit our specification of the curve $C$ to a small neighbourhood of the origin $(\theta, y)=(0,0)$. The left out portion despite being significantly larger has no consequence on the evaluation of (4.8). Hence, it suffices to take $\theta \sim A y^{b}$ to be the asymptotic form that any general parametric representation of $C$ would assume in the extreme vicinity of the rear stagnation point. An equivalent representation in the wake coordinates reads as $z \sim A x^{b}$ and since the wake solution holds only downstream of the rear stagnation point, one may impose constraints $A>0$ and $b>1 / 2$.

We substitute the asymptotic form of $C$ in (4.8), while simultaneously substituting for the boundary layer and wake solutions (3.13) and (3.25), respectively. After some rather lengthy manipulations that are described in full detail in Appendix $\mathrm{C}$, we eventually obtain

$$
\begin{aligned}
& \frac{2 \mu}{\rho U_{\infty}^{3} a} \int_{\Omega_{u}} \omega_{b l, w}^{2} \mathrm{~d} \Omega \approx \frac{4 \sqrt{2}}{R e^{3 / 2}}\left(\int_{\theta=0}^{\pi} \int_{y^{*}=0}^{\frac{1}{\delta}\left(\frac{\theta}{A}\right)^{1 / b}} \omega_{b l}^{* 2} \mathrm{~d} y^{*} \mathrm{~d} \theta+\int_{x=0}^{\infty} \int_{z^{*}=0}^{\frac{A x^{b}}{\delta}} \omega_{w}^{* 2} \mathrm{~d} z^{*} \mathrm{~d} x\right) \\
& \approx \frac{63.68+73.56 b-23.69 \ln \left(A \delta^{b}\right)}{(b+1) \operatorname{Re} 3 / 2}+\frac{160.55+150.67 b+23.69 \ln (A / \delta)}{(b+1) R e^{3 / 2}} \\
& =\frac{216.02+11.85 \ln R e}{R e^{3 / 2}},
\end{aligned}
$$

where only the leading-order terms have been retained. Note that the above result is independent of the constants $A$ and $b$ and, therefore, the shape of the dividing curve $C$, as asserted previously. This independence is not surprising since in our estimation of (4.8), 


\section{High-Reynolds-number flow past a shear-free cylinder}

we essentially construct a single uniformly valid vorticity field from the boundary layer and the wake region solutions. The independence is consistent with the general expectation from the procedure of obtaining solutions using matched asymptotic expansions (Van Dyke 1975). Specifically, the leading-order term associated with any quantity derivable from the matched asymptotic expansion is independent of the way in which solutions in distinct regions that share common regions of validity are combined to form a composite solution.

Next we evaluate the integral (II). Recasting (II) in terms of the non-dimensional quantities we obtain

$$
\frac{2 \mu}{\rho U_{\infty}^{3} a} \int_{\Omega_{s}}\left(\omega_{s}^{2}-\lim _{\theta \rightarrow 0} \omega_{b l}^{2}\right) \mathrm{d} \Omega=\frac{4 \sqrt{2}}{R e^{3 / 2}} \int_{\theta_{s}^{*}=0}^{\infty} \int_{y_{s}^{*}=0}^{\infty}\left(\omega_{s}^{* 2}-\lim _{\theta \rightarrow 0} \omega_{b l}^{* 2}\right) \mathrm{d} y_{s}^{*} \mathrm{~d} \theta_{s}^{*} .
$$

Numerical evaluation of the above integral yields

$$
\frac{2 \mu}{\rho U_{\infty}^{3} a} \int_{\Omega_{s}}\left(\omega_{s}^{2}-\lim _{\theta \rightarrow 0} \omega_{b l}^{2}\right) \mathrm{d} \Omega \approx \frac{5.45}{R e^{3 / 2}} .
$$

Combining the relationships (4.5), (4.9) and (4.11) we arrive at the following expression for the drag coefficient:

$$
C_{D}^{(2)}=\frac{16 \pi}{\operatorname{Re}}\left(1+\frac{0.24 \ln R e-1.79}{\sqrt{\operatorname{Re}}}\right) .
$$

Here the superscript '(2)' indicates the order to which the terms in the asymptotic expansion are retained. For comparison purposes, we define a scaled correction term $\tilde{C}_{D}$ by eliminating the contribution from the first-order term in the asymptotic expansion of the drag coefficient $C_{D}^{(1)}$ (or, equivalently, the power loss coefficient associated with the inviscid potential flow) and subsequently normalizing with respect to $R e^{-3 / 2}$, i.e.

$$
\tilde{C}_{D}=\operatorname{Re}^{3 / 2}\left(C_{D}-C_{D}^{(1)}\right) .
$$

The scaled correction term $\tilde{C}_{D}$ quantifies the contributions to the drag coefficient from the second and any higher-order terms in the asymptotic expansion of the viscous corrections to the potential flow velocity field. Combining (4.12) and (4.13) we deduce that

$$
\tilde{C}_{D}^{(2)}=11.85 \ln R e-90.17
$$

for the scaled correction corresponding to the second-order estimate of the drag coefficient (4.12).

Our asymptotic estimates can be directly compared with the predictions from high-resolution DNS. Figure 3 depicts a comparison of the theoretical result (4.12) for the drag coefficient with the predictions from DNS over more than three decades of variation in the Reynolds number. The theoretical result (4.12) is in excellent agreement with the DNS predictions, the agreement being near perfect over the range $R e \gtrsim 500$. With a progressive increase in the Reynolds number, the $O\left(R e^{-3 / 2}\right)$ term in (4.12) diminishes at a rate that is higher than the leading-order $O\left(R e^{-1}\right)$ term. The influence of inclusion of the $O\left(R e^{-3 / 2}\right)$ term in the theoretical estimate for drag coefficient is therefore expected to be most prominent over the low Reynolds numbers range. This is clearly evident from the trends depicted in figure 3. Specifically, compared with (4.3), the asymptotic 
A. Kumar, N.M.A. Rehman, P. Giri and R.K. Shukla

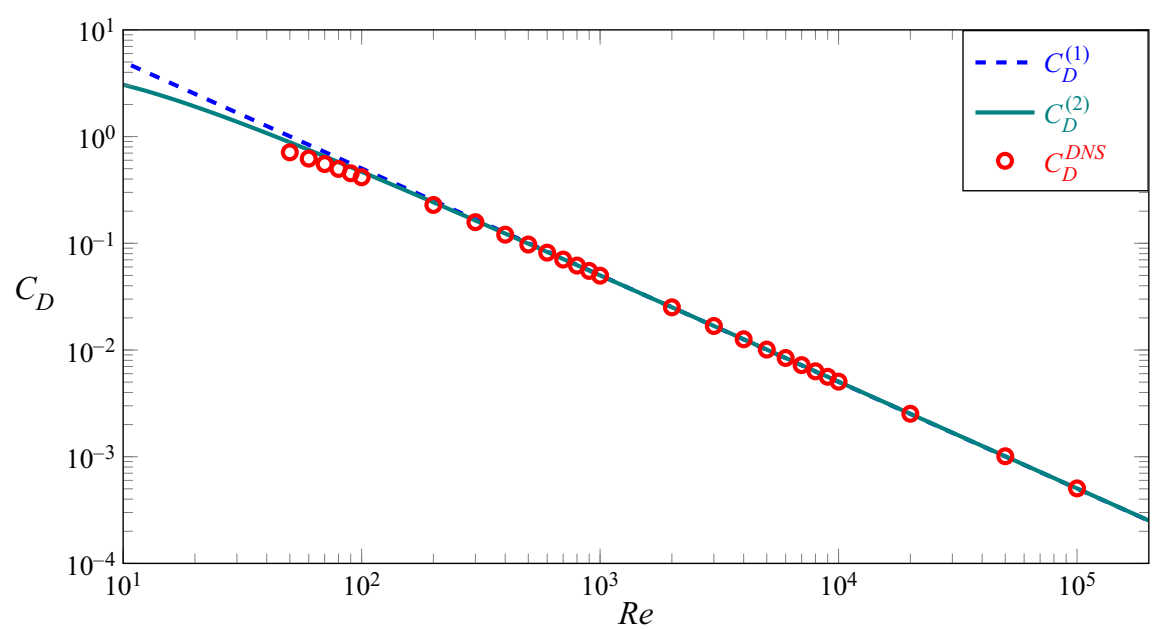

Figure 3. Drag coefficient $C_{D}$ as a function of the Reynolds number. Dashed lines labelled $C_{D}^{(1)}$ and solid lines labelled $C_{D}^{(2)}$ represent theoretical estimates given by (4.3) and (4.12), respectively. Open circles labelled $C_{D}^{D N S}$ indicate results obtained from DNS.

result (4.12) for $C_{D}^{(2)}$ is in appreciably better agreement with the DNS predictions over the range $R e \lesssim 100$. Beyond $R e \approx 500$, the asymptotic estimates (4.12) and (4.3) are indistinguishable and crucially, in remarkable agreement with the DNS predictions.

To assess the significance of the higher-order $O\left(R^{-3 / 2}\right)$ term in the theoretical estimate (4.12), we compare the scaled correction term (4.13) deduced from the analysis with the one obtained from DNS results. Figure 4 illustrates this comparison over the full range encompassing over three orders of magnitude variation in the Reynolds number. By definition, $\tilde{C}_{D}^{(1)}=0$ irrespective of $R e$, as shown using blue dotted lines in figure 4 . A comparison between the scaled correction given by (4.14) with the one computed from DNS indicates significant deviation over the low-Reynolds-number range $R e \lesssim 1000$. For $R e \gtrsim 2000$, the assumptions inherent in our asymptotic analysis $\left(R e^{-1 / 2} \ll 1\right.$ and $\left.R e^{-1 / 4} \underset{\ll}{\ll}\right)$ are met and the scaled correction $\tilde{C}_{D}^{(2)}$ exhibits remarkable agreement with the predictions from DNS. Importantly, except over a small range of Reynolds number centred around $R e \sim 2000$, compared with $\tilde{C}_{D}^{(1)}, \tilde{C}_{D}^{(2)}$ is consistently in better agreement with the scaled correction $\tilde{C}_{D}^{D N S}$ estimated from the detailed simulations. Thus, the inclusion of $O\left(R^{-3 / 2}\right)$ term improves the asymptotic analysis based prediction of the drag coefficient across the entire range of Reynolds number.

The scaled correction $\tilde{C}_{D}^{(2)}$ is unusual in that it explicitly depends on a logarithmic term in the Reynolds number. This peculiar dependence is specific to the case of a shear-free circular cylinder and is not observed in the case of a shear-free sphere (Moore 1963). The dependence leads to an inversion in the sign of the scaled correction $\tilde{C}_{D}^{(2)}$ at a critical Reynolds number $R e_{c} \approx 2017$. The $O\left(R e^{-3 / 2}\right)$ term thus reduces the drag coefficient over the range $R e<R e_{c}$, while increasing it for $R e>R e_{c}$. The foregoing observation is supported by the DNS results shown in figure 4, with the simulations indicating a higher critical Reynolds number of about 2340 for an inversion in the sign of the contribution from the higher-order terms. 


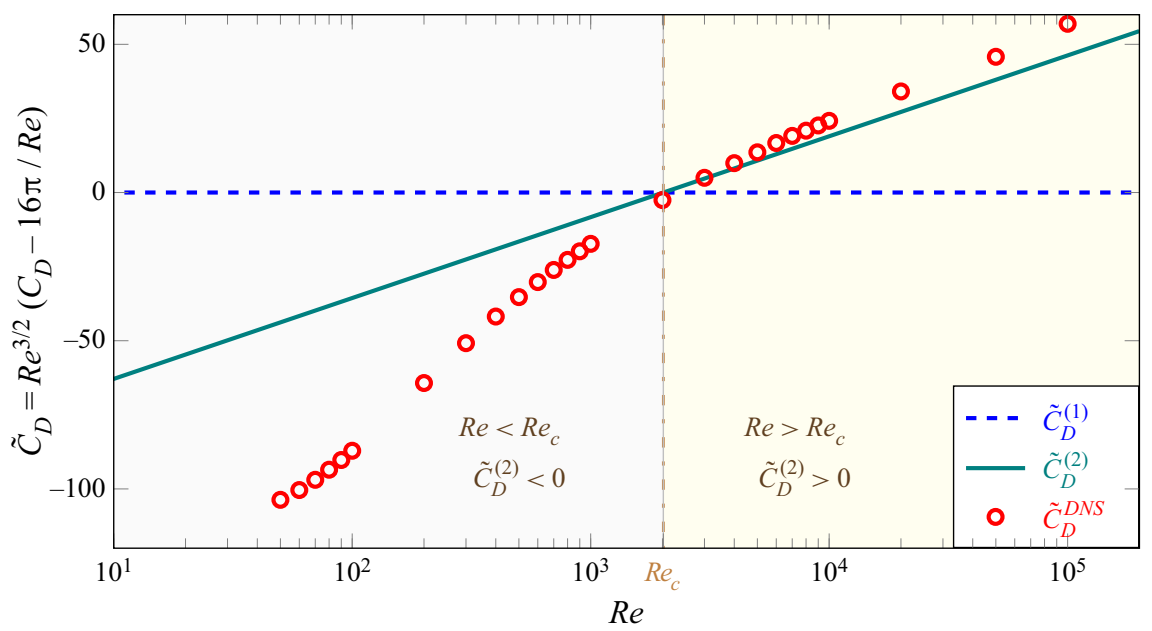

Figure 4. The scaled correction to the drag coefficient $\tilde{C}_{D}$ as a function of the Reynolds number. Dashed lines labelled $\tilde{C}_{D}^{(1)}$ and solid lines labelled $\tilde{C}_{D}^{(2)}$ represent scaled corrections associated with the theoretical estimates (4.3) and (4.12), respectively. Open circles labelled $\tilde{C}_{D}^{D N S}$ indicate scaled correction for the drag coefficient computed from DNS. The vertical dash-dotted line indicates the critical Reynolds number $\operatorname{Re}_{c}$ below and above which $\tilde{C}_{D}^{(2)}<0$ (shown as the light grey shaded region) and $\tilde{C}_{D}^{(2)}>0$ (shown as the light yellow shaded region), respectively.

The aforementioned inversion in the sign of high-order contributions has important energetic implications. Specifically, our results indicate that over the low-Reynolds-number range $R e<R e_{c}$, the net frictional loss associated with the irrotational potential flow overwhelms the net loss associated with flow past a shear-free circular cylinder. For $R e>R e_{c}$, the net frictional loss associated with the irrotational potential flow is marginally lower than the loss associated with the shear-free cylinder boundary, with the difference between the two diminishing progressively with the Reynolds number. In light of the previous findings of Shukla \& Arakeri (2013), we therefore conclude that the tangential surface velocities corresponding to the viscous potential flow and a shear-free condition minimize frictional loss associated with flow past an impermeable circular cylinder at large $\left(R e>R e_{c}\right)$ and small $\left(R e<R e_{c}\right)$ Reynolds numbers, respectively. In stark contrast, Moore's asymptotic analysis (Moore 1963) for an axisymmetric spherical configuration suggests that the net power loss associated with the potential flow overwhelms the loss associated with a shear-free boundary, irrespective of the Reynolds number.

Our approach of finding the net dissipation may seem overly complicated, especially in view of the comparatively simpler analysis involved in the estimation of drag on a shear-free sphere (Moore 1963). The apparent complication however is merely due to the disparity in the contribution from the rear stagnation region in the planar and axisymmetric configurations. In an axisymmetric spherical set-up, the $O\left(R e^{-11 / 6}\right)$ contribution to the dissipation from the rear stagnation region is dwarfed by the $O\left(R e^{-3 / 2}\right)$ contribution from the wake and boundary layer regions. A neglect of the contribution from the rear stagnation region and a utilization of the boundary layer and wake region solutions over the entire flow domain are both warranted in an analysis of the $O\left(R e^{-3 / 2}\right)$ term in the asymptotic expansion of the drag coefficient. Such a simplification is not possible for a planar cylindrical configuration as all the three wake, boundary layer and rear stagnation regions contribute to an equal order to the net frictional loss. In our analysis of the 


\section{A. Kumar, N.M.A. Rehman, P. Giri and R.K. Shukla}

contribution from the rear stagnation region, an additional complication arises from the nonlinear coupling between the correctional and inviscid base state flow variables, with the equality in their order of magnitudes eliminating the possibility of a linearization based simplification.

\section{Summary}

To conclude, we developed an asymptotic theory for the high-Reynolds-number flow past a shear-free circular cylinder. The simplest predictive theory for this flow, namely the classical irrotational potential flow theory, suffers from D'Alembert's paradox as it predicts a fore-aft symmetric stress field for which the hydrodynamic resistance experienced by the shear-free cylinder must necessarily vanish. Attributing this paradoxical conclusion to a violation of the perfect slip boundary condition on the shear-free cylinder surface, we introduced viscous correctional terms to the irrotational potential flow that account for both the finite vorticity production over the shear-free cylinder surface and its highly efficient convection into the wake region. We combined interdependent perturbation expansion based analysis in each of the distinct boundary layer, rear stagnation and wake regions over which vorticity is first produced and subsequently convected downstream.

We demonstrated that a neglect of the relatively insignificant streamwise gradients in a thin region surrounding the cylinder surface leads to a linear boundary layer equation for the most significant leading-order term in the perturbation expansion of the viscous correction to the inviscid base flow. Using a similarity-transformation-induced dimension reduction, we derived a single-parameter-dependent family of distinct solutions to the characteristic-scale-deficient boundary layer equation. Quite atypically however, none of these solutions were entirely compatible with all the necessary boundary conditions. Exploiting the linearity of the boundary layer equation, we developed an unconventional all-boundary-condition-compatible solution that instead of a single self-similar term consisted of a weighted superposition of countably infinite members from the family of self-similar solutions.

The solution to the boundary layer equation grows unbounded in the vicinity of the rear stagnation region. We attributed this unphysical behaviour to a breakdown of the principal assumption of streamwise gradients being negligibly small compared with the surface-normal gradients. Through a careful re-examination of the governing equations we arrived at the characteristic scales appropriate for the rear stagnation region. We showed that in an $O\left(R e^{-1 / 4}\right)$ sized region around the rear stagnation point, the non-dimensional velocity fields for both the inviscid base state and the first-order term in the correctional perturbation expansion are of comparable $O\left(R e^{-1 / 4}\right)$ magnitude and crucially, are both governed by inviscid dynamics. We noted that the foregoing observation on the magnitude of base state and first-order correctional terms contrasted sharply with Moore's analysis on axisymmetric flow past a shear-free sphere (Moore 1965), but, was consistent with the analysis of Harper (1963) for planar stagnation point flows. We derived an elliptic partial integro-differential equation with a non-local source term for the correctional velocity induced distortion in the streamfunction associated with the inviscid rotational flow over the rear stagnation region. Numerical solution of the analytically intractable nonlinear partial integro-differential equation allowed us to determine well-behaved, bounded correctional fields that, in an overlap region, were consistent with the boundary layer solution. Comparisons with DNS showed our theoretical predictions of the first-order correctional term in the tangential surface velocity to be in excellent agreement with the high-resolution computations, the agreement being particularly outstanding at a 


\section{High-Reynolds-number flow past a shear-free cylinder}

high Reynolds number of $10^{5}$, which is when the critical assumptions $R e^{-1 / 2} \ll 1$ and $R e^{-1 / 4} \ll 1$ associated with the boundary layer and rear stagnation analyses are both satisfied.

Our analysis of the wake region centred around simplifying assumptions similar to those associated with our boundary layer analysis. We showed that the high-Reynolds-number vorticity transport through the narrow wake region is essentially governed by a linear parabolic advection-diffusion equation. We derived an explicit closed-form solution that, like the boundary layer solution, consisted of a weighted superposition of infinite self-similar wake profiles originating at distinct locations along the axis of symmetry. We demonstrated that sufficiently far downstream our infinite-term wake solution desirably simplified to the well-known asymptotic form for a planar wake associated with a two-dimensional body, the drag coefficient for which precisely equals the leading-order estimate of $16 / R e$ for a shear-free circular cylinder.

Utilizing the equivalence between the energy dissipation rate and the power expended in overcoming the drag force, we derived explicit expressions for the first- and second-order terms in the asymptotic expansion of the drag coefficient. Our theoretical estimate of the drag coefficient was in agreement with the predictions from an exhaustive set of DNS spanning over three decades of order of magnitude variation in the Reynolds number. Importantly, we demonstrated that the second-order term's atypical logarithmic dependence on the Reynolds number, predicted from our theoretical analysis, was in remarkable agreement with the results from high Reynolds number $(R e \gtrsim 2000)$ DNS. The unusual logarithmic dependence led to identification of a critical Reynolds number only below which the energy dissipation rate (power loss coefficient) for the irrotational potential flow exceeds the dissipation rate for flow past a shear-free cylinder.

To the best of our knowledge, our asymptotic analysis provides the first theoretical prediction and quantitative assessment of the finite-viscosity effects in the high-Reynolds-number flow past a shear-free circular cylinder. The viscosity-induced modifications to the inviscid base flow predicted from our analysis are far less pronounced than for flow past a no-slip cylindrical surface. Nonetheless, the implications of inclusion of finite-viscosity effects in our theoretical analysis are quite significant. Our results and analysis pave a way towards theoretical investigations on the spatiotemporal stability of perturbation-sensitive flow features such as boundary layers and wakes. An understanding of the susceptibility of these instability-prone flow features to external perturbations and an assessment of their potential to destabilize the free-shear-enabling mechanism itself (as in the case of superhydrophobic surfaces for instance) will be crucial in technological applications that rely on sustained slip over prolonged periods. Our analysis could form the basis of theoretical investigations of flows over asymmetric shear-free boundaries with non-constant curvature. The curvature-driven increase in vorticity production could enhance the Reynolds-number-dependent contrast in viscous correctional term's contribution to the net dissipation in such flows. This would bear important implications with regard to the optimality of a perfect slip condition in minimizing dissipation associated with flows past streamlined and bluff bodies.

Acknowledgements. The authors acknowledge support received from the NVIDIA Corporation (hardware donation program) and Supercomputing Education and Research Center-Indian Institute of Science (runtime on Cray XC40).

Declaration of interest. The authors report no conflict of interest.

Funding. R.K.S. acknowledges support received from the Department of Science and Technology (DSTO 1329). 


\section{A. Kumar, N.M.A. Rehman, P. Giri and R.K. Shukla}

\section{Author ORCIDs.}

(1) Anuj Kumar https://orcid.org/0000-0002-9203-9177;

(ㄱ) Ratnesh K. Shukla https://orcid.org/0000-0001-9169-2070.

\section{Appendix A. Solution of the governing equation for $\tilde{u}_{\phi}^{*}$,}

Here we outline our procedure of determining the non-dimensional correction velocity component along the circumferential direction. The governing boundary layer equation (3.7) admits a similarity solution. To show this, we set $\tilde{u}_{\phi}^{*}=H(\phi) F(\eta)$, where $\eta=$ $y^{*} / g(\phi)$. Substitution of the above form in (3.7) yields

$$
F^{\prime \prime}+2 \eta F^{\prime}\left(g^{2} \cos \phi+g \frac{\mathrm{d} g}{\mathrm{~d} \phi} \sin \phi\right)-2 F\left(g^{2} \cos \phi+g^{2} \sin \phi \frac{1}{H} \frac{\mathrm{d} H}{\mathrm{~d} \phi}\right)=0 .
$$

Self-similarity is achieved when the terms inside the brackets are constants independent of $\phi$. Without loss of generality, we set

$$
2 g^{2} \cos \phi+2 g \frac{\mathrm{d} g}{\mathrm{~d} \phi} \sin \phi=1, \quad 2 g^{2} \cos \phi+2 g^{2} \sin \phi \frac{1}{H} \frac{\mathrm{d} H}{\mathrm{~d} \phi}=\alpha,
$$

where $\alpha$ is a constant. The only solution of (A2) that is also a solution of (3.7) and simultaneously satisfies the boundary condition $(3.8 a)$ is

$$
g(\phi)=\frac{1}{\sqrt{2} \cos \frac{\phi}{2}}, \quad H(\phi)=\frac{\beta\left(\sin \frac{\phi}{2}\right)^{\alpha}}{\sin \phi},
$$

where $\beta$ is a constant and $\alpha>1$. The above functional forms of $g(\phi)$ and $H(\phi)$ yield

$$
\frac{\partial \tilde{u}_{\phi}^{*}}{\partial y^{*}}=\sqrt{2} \beta F^{\prime}(0)\left(\sin \frac{\phi}{2}\right)^{\alpha-1}, \quad \text { at } y^{*}=0 .
$$

Clearly the above expression cannot be matched with the boundary condition (3.8c) for any choice of parameter $\beta$.

The linearity of the governing equation (3.7) can be exploited to make further progress. To this end, we set $\alpha=2 n+2, \forall n \geqslant 0, n \in \mathbb{Z}$ and $\beta=\sqrt{2} / F_{n}^{\prime}(0)$ so that the boundary condition (A4) assumes the form

$$
\frac{\partial \tilde{u}_{\phi}^{*}}{\partial y^{*}}=\left(\sin \frac{\phi}{2}\right)^{2 n+1}, \quad \text { at } y^{*}=0 .
$$

For the above choice of parameters, (A1) simplifies as

$$
F_{n}^{\prime \prime}+\eta F_{n}^{\prime}-(2 n+2) F_{n}=0
$$

where a subscript $n$ is used to denote the $n$-specific solution. Likewise $\tilde{u}_{\phi, n}^{*}=H_{n}(\phi) F_{n}(\eta)$ and the boundary condition $(3.8 b)$ transforms into

$$
F_{n}(\eta) \rightarrow 0 \quad \text { as } \eta \rightarrow \infty .
$$

The solution of (A6) that satisfies the boundary condition (A7) is readily found,

$$
F_{n}(\eta)=\int_{0}^{\pi} \frac{1}{\sqrt{2 \pi}} \exp \left(-\frac{\eta^{2}}{2 \cos ^{2}\left(\frac{\alpha}{2}\right)}\right) \sin ^{2 n+2}\left(\frac{\alpha}{2}\right) \mathrm{d} \alpha, \quad \forall n \geqslant-1 \text { and } n \in \mathbb{Z} .
$$


To establish that (A8) is indeed a solution of (A1), we first consider the specific case of $n=-1$,

$$
\begin{aligned}
F_{-1}(\eta) & =\int_{0}^{\pi} \frac{1}{\sqrt{2 \pi}} \exp \left(-\frac{\eta^{2}}{2 \cos ^{2}\left(\frac{\alpha}{2}\right)}\right) \mathrm{d} \alpha \\
& =\int_{\eta}^{\infty} \exp \left(-\frac{s^{2}}{2}\right)\left(\int_{0}^{\pi} \frac{s}{\sqrt{2 \pi} \cos ^{2}\left(\frac{\alpha}{2}\right)} \exp \left(\frac{-s^{2} \tan ^{2}\left(\frac{\alpha}{2}\right)}{2}\right) \mathrm{d} \alpha\right) \mathrm{d} s \\
& =\left.\int_{\eta}^{\infty} \exp \left(-\frac{s^{2}}{2}\right) \operatorname{erf}\left(\frac{s \tan \left(\frac{\alpha}{2}\right)}{\sqrt{2}}\right)\right|_{\alpha=0} ^{\pi} \mathrm{d} s=\sqrt{\frac{\pi}{2}} \operatorname{erfc}\left(\frac{\eta}{\sqrt{2}}\right) .
\end{aligned}
$$

which indeed is the solution of (A6) for $n=-1$. The sequence of steps listed in (A9) may in fact be considered a simple derivation of the well-known Craig's formula (Craig 1991), the first expression on the right-hand side of (A9) being another form of the Gaussian distribution function.

Next we show that the following relationship holds between $F_{n+1}(\eta)$ and $F_{n}(\eta)$ :

$$
\begin{aligned}
F_{n+1}(\eta)= & \int_{0}^{\pi} \frac{1}{\sqrt{2 \pi}} \exp \left(-\frac{\eta^{2}}{2 \cos ^{2}\left(\frac{\alpha}{2}\right)}\right) \sin ^{2 n+4}\left(\frac{\alpha}{2}\right) \mathrm{d} \alpha \\
= & \left.\int_{\eta}^{\infty}\left\{\int_{0}^{\pi} \frac{\eta_{1}}{\sqrt{2 \pi} \cos ^{2}\left(\frac{\alpha}{2}\right)} \exp -\frac{\eta_{1}^{2}}{2 \cos ^{2}\left(\frac{\alpha}{2}\right)}\right) \sin ^{2 n+4}\left(\frac{\alpha}{2}\right) \mathrm{d} \alpha\right\} \mathrm{d} \eta_{1} \\
= & \int_{\eta}^{\infty}\left\{-\left.\operatorname{erfc}\left(\frac{\eta_{1}}{\sqrt{2} \cos \left(\frac{\alpha}{2}\right)}\right) \sin ^{2 n+3}\left(\frac{\alpha}{2}\right)\right|_{0} ^{\pi}\right. \\
& \left.+\frac{2 n+3}{2} \int_{0}^{\pi} \operatorname{erfc}\left(\frac{\eta_{1}}{\sqrt{2} \cos \left(\frac{\alpha}{2}\right)}\right) \sin ^{2 n+2}\left(\frac{\alpha}{2}\right) \cos \left(\frac{\alpha}{2}\right) \mathrm{d} \alpha\right\} \mathrm{d} \eta_{1} \\
= & (2 n+3) \int_{\eta}^{\infty} \int_{\eta_{1}}^{\infty}\left\{\int_{0}^{\pi} \frac{1}{\sqrt{2 \pi}} \exp \left(-\frac{\eta_{2}^{2}}{2 \cos ^{2}\left(\frac{\alpha}{2}\right)}\right) \sin ^{2 n+2}\left(\frac{\alpha}{2}\right) \mathrm{d} \alpha\right\} \mathrm{d} \eta_{2} \mathrm{~d} \eta_{1} \\
= & (2 n+3) \int_{\eta}^{\infty} \int_{\eta_{1}}^{\infty} F_{n}\left(\eta_{2}\right) \mathrm{d} \eta_{2} \mathrm{~d} \eta_{1} \cdot
\end{aligned}
$$

Consequently,

$$
F_{n+1}^{\prime}(\eta)=-(2 n+3) \int_{\eta}^{\infty} F_{n}(z) \mathrm{d} z \quad \text { and } \quad F_{n+1}^{\prime \prime}(\eta)=(2 n+3) F_{n}(\eta) . \quad(\mathrm{A} 11 a, b)
$$




\section{A. Kumar, N.M.A. Rehman, P. Giri and R.K. Shukla}

Integrating the expression (A6) twice we obtain

$$
F_{n}(\eta)-\eta \int_{\eta}^{\infty} F_{n}\left(\eta_{1}\right) \mathrm{d} \eta_{1}-\int_{\eta}^{\infty} \int_{\eta_{1}}^{\infty}(2 n+4) F_{n}\left(\eta_{2}\right) \mathrm{d} \eta_{2} \mathrm{~d} \eta_{1}=0 .
$$

Using relationships (A10), (A11) and (A12), one finds that if (A8) is the solution of (A6) for some integer $n$ then the result holds for $n+1$ as well. The proof therefore follows from induction.

We next determine a solution to (3.7) that satisfies all the three boundary conditions $(3.8 a-c)$. We begin with an evaluation of $F_{n}^{\prime}(0)$ :

$$
F_{n}^{\prime}(0)=\left.\int_{0}^{\pi} \frac{-\eta}{\sqrt{2 \pi} \cos ^{2}\left(\frac{\alpha}{2}\right)} \exp \left(-\frac{\eta^{2}}{2 \cos ^{2}\left(\frac{\alpha}{2}\right)}\right) \sin ^{2 n+2}\left(\frac{\alpha}{2}\right) \mathrm{d} \alpha\right|_{\eta=0} .
$$

Since the above integrand is singular at $\eta=0$, we first simplify (A13) using integration by parts and subsequently apply the limit $\eta=0$ to the resulting well-behaved integrand, i.e.

$$
\begin{aligned}
F_{n}^{\prime}(0) & =-\left.\frac{2 n+1}{2} \int_{0}^{\pi} \operatorname{erfc}\left(\frac{\eta}{\sqrt{2} \cos \left(\frac{\alpha}{2}\right)}\right) \sin ^{2 n}\left(\frac{\alpha}{2}\right) \cos \left(\frac{\alpha}{2}\right) \mathrm{d} \alpha\right|_{\eta=0} \\
& =-\frac{2 n+1}{2} \mathrm{~B}\left(\frac{2 n+1}{2}, 1\right)=-1,
\end{aligned}
$$

here $\mathrm{B}(x, y)$ denotes the beta function.

Next, recasting the right-hand side of the boundary condition (3.8c) using (3.11) and superimposing the similarity solutions $\tilde{u}_{\phi, n}^{*}$ using the weights in (3.11), we obtain the solution of the scaled azimuthal correction velocity component $\left(\tilde{u}_{\phi}^{*}\right)$ as

$$
\begin{aligned}
\tilde{u}_{\phi}^{*} & =\sum_{n=0}^{\infty} w_{n} \tilde{u}_{\phi, n}^{*}=\sum_{n=0}^{\infty} w_{n} H_{n}(\phi) F_{n}(\eta) \\
& =\frac{-4}{\sqrt{\pi}} \tan \left(\frac{\phi}{2}\right) \int_{0}^{\pi} \exp \left(\frac{-\eta^{2}}{2 \cos ^{2}\left(\frac{\alpha}{2}\right)}\right) \sin ^{2}\left(\frac{\alpha}{2}\right) \sqrt{1-\sin ^{2}\left(\frac{\alpha}{2}\right) \sin ^{2}\left(\frac{\phi}{2}\right)} \mathrm{d} \alpha,
\end{aligned}
$$

where $\eta=\sqrt{2} y^{*} \cos (\phi / 2)$.

\section{Appendix B. Numerical solution of the non-dimensional distortion flow streamfunction (3.19)}

For computational convenience, we first apply the following transformation to the governing equation (3.19) for the non-dimensional distortion flow streamfunction $\tilde{\psi}_{S}^{*}$ :

$$
\sigma=\theta_{s}^{*} y_{s}^{*}, \quad \tau=\left(\theta_{s}^{* 2}-y_{s}^{* 2}\right) / 2 .
$$




\section{High-Reynolds-number flow past a shear-free cylinder}

Rewritten in $(\sigma, \tau)$ coordinates, (3.19) assumes the form

$$
\left(\frac{\partial^{2} \tilde{\psi}_{s}^{*}}{\partial \sigma^{2}}+\frac{\partial^{2} \tilde{\psi}_{s}^{*}}{\partial \tau^{2}}\right)=\frac{1}{\sqrt{\pi}} \int_{0}^{\pi} \frac{\tilde{\psi}_{s}^{*}+2 \sigma}{\left(\sigma^{2}+\tau^{2}\right)^{1 / 2}} \exp \left(\frac{-\left(\tilde{\psi}_{s}^{*}+2 \sigma\right)^{2}}{16 \cos ^{2}\left(\frac{\alpha}{2}\right)}\right) \frac{\sin ^{2}\left(\frac{\alpha}{2}\right)}{\cos \left(\frac{\alpha}{2}\right)} \mathrm{d} \alpha
$$

while the boundary conditions $(3.20 a-c)$ transform as follows:

$\tilde{\psi}_{s}^{*}=0$ at $\sigma=0, \quad \frac{\partial \tilde{\psi}_{s}^{*}}{\partial \sigma}=0 \quad$ as $\sigma \rightarrow \infty, \quad \frac{\partial \tilde{\psi}_{s}^{*}}{\partial \tau}=0 \quad$ at $\tau=0 \quad$ and $\quad$ as $\tau \rightarrow \infty$.

The boundary conditions in (B3) implying impermeability, uniformity of the streamfunction outside the thin stagnation layer, symmetry of the flow about the line $\theta_{s}^{*}=y_{s}^{*}$ and unidirectionality of the flow towards the end stage of the boundary layer, respectively.

To solve (B2), we employ an iterative process. We begin with a uniform initial guess state corresponding to $\tilde{\psi}_{s}^{*}=0$ everywhere. Subsequently, in each iteration we use the present known $\tilde{\psi}_{s}^{*}$ to compute the right-hand side of (B2). With this known right-hand side we solve the Poisson equation for $\tilde{\psi}_{s}^{*}$ along with boundary conditions (B3). We repeat this iterative process until convergence is achieved (the maximum pointwise difference in $\tilde{\psi}_{s}^{*}$ between successive iterations is less than $10^{-10}$ ).

To discretize the Poisson equation $\tilde{\psi}_{s}^{*}$, we employ standard second-order central finite differences on exponentially stretched grids along both $\sigma$ and $\tau$ directions. Exponential stretching enables solution adaptivity so that the resulting spatial resolution is the highest in the regions associated with large vorticity gradients. To solve for the vector of unknowns consisting of discrete pointwise $\tilde{\psi}_{s}^{*}$, we employ a specialized Gaussian elimination method that exploits the sparsity of the system to reduce the overall computational expense.

To determine the tangential surface velocity at the shear-free cylindrical boundary, we evaluate the relationship

$$
\tilde{u}_{\theta_{(s)}^{*}}^{*}=-\left.\frac{\partial \tilde{\psi}_{s}^{*}}{\partial y_{s}^{*}}\right|_{y_{s}^{*}=0}=-\left.\sqrt{2 \tau} \frac{\partial \tilde{\psi}_{s}^{*}}{\partial \sigma}\right|_{\sigma=0}
$$

using a sixth-order one-sided finite difference approximation for the first derivative. The vorticity in the stagnation region is conveniently computed from the converged $\tilde{\psi}_{S}^{*}$ via the following transformed variant of the relationship (3.19):

$$
\omega_{s}^{*}=-\frac{2}{\sqrt{\pi}} \int_{0}^{\pi}\left(\tilde{\psi}_{s}^{*}+2 \sigma\right) \exp \left(\frac{-\left(\tilde{\psi}_{s}^{*}+2 \sigma\right)^{2}}{16 \cos ^{2}\left(\frac{\alpha}{2}\right)}\right) \frac{\sin ^{2}\left(\frac{\alpha}{2}\right)}{\cos \left(\frac{\alpha}{2}\right)} \mathrm{d} \alpha .
$$

Likewise, in $(\sigma, \tau)$ coordinates, the integral in the expression (4.10) assumes the following form:

$$
\int_{\theta_{s}^{*}=0}^{\infty} \int_{y_{s}^{*}=0}^{\infty}\left(\omega_{s}^{* 2}-\left.\omega_{b l}^{* 2}\right|_{\theta \rightarrow 0}\right) \mathrm{d} y_{s}^{*} \mathrm{~d} \theta_{s}^{*}=2 \int_{\sigma=0}^{\infty} \int_{\tau=0}^{\infty} \frac{\left(\omega_{s}^{* 2}-\left.\omega_{b l}^{* 2}\right|_{\theta \rightarrow 0}\right)}{2\left(\sigma^{2}+\tau^{2}\right)^{1 / 2}} \mathrm{~d} \sigma \mathrm{d} \tau .
$$

To evaluate this integral numerically, we apply a two-dimensional generalization of the Simpson's quadrature rule. 


\section{A. Kumar, N.M.A. Rehman, P. Giri and R.K. Shukla}

\section{Appendix C. Derivation of the estimate (4.9)}

To establish the result (4.9), we first consider evaluation of the leading-order term in the integral

$$
\mathrm{I}\left(A, n, s_{f}, \delta, M(s), N(s)\right)=\int_{s=0}^{s_{f}} s^{2} M(s) \int_{t=0}^{A s^{n} / \delta} t^{2} \exp \left(-s^{2} t^{2} N^{2}(s)\right) \mathrm{d} t \mathrm{~d} s,
$$

in the limit of $\delta \rightarrow 0^{+}$, where $n>0, A>0, N(s)>0, \forall s \in\left[0, s_{f}\right]$. Subsequently, we show that (4.9) is a special case of the general integral (C1).

We denote the limiting expressions

$$
M(s) \rightarrow M_{0}, \frac{\mathrm{d} M(s)}{\mathrm{d} s} \rightarrow M_{0}^{\prime}, N(s) \rightarrow N_{0}, \text { and } \frac{\mathrm{d} N(s)}{\mathrm{d} s} \rightarrow N_{0}^{\prime} \text { as } s \rightarrow 0,
$$

where $M_{0}, N_{0}, M_{0}^{\prime}$ and $N_{0}^{\prime}$ are all bounded. Performing the integration with respect to $t$ in (C1) yields

$$
\mathrm{I}=\int_{0}^{s_{f}} \frac{A M(s)}{4 s N^{2}(s)}\left[\frac{-2 s^{n+1}}{\delta} \exp \left(\frac{-s^{2 n+2} A^{2} N^{2}(s)}{\delta^{2}}\right)+\frac{\sqrt{\pi}}{A N(s)} \operatorname{erf}\left(\frac{s^{n+1} A N(s)}{\delta}\right)\right] \mathrm{d} s .
$$

We split the above integral into two parts with limits of integration in the first and second parts ranging from $s=0$ to $s=\epsilon$ and $s=\epsilon$ to $s=s_{f}$, respectively. We set $\epsilon$ such that $\delta^{1 /(n+1)} \ll \epsilon$ and $\epsilon \ll 1$. This choice ensures that the integrand of the first part can be simplified by using the limit $\epsilon \rightarrow 0$, while the integrand in the second part can be simplified by taking the limit $\epsilon^{n+1} / \delta \rightarrow \infty$.

Simplification of the first part proceeds as follows:

$$
\begin{aligned}
\mathrm{I}_{1}= & \int_{0}^{\epsilon} \frac{A M(s)}{4 s N^{2}(s)}\left[\frac{-2 s^{n+1}}{\delta} \exp \left(\frac{-s^{2 n+2} A^{2} N^{2}(s)}{\delta^{2}}\right)+\frac{\sqrt{\pi}}{A N(s)} \operatorname{erf}\left(\frac{s^{n+1} A N(s)}{\delta}\right)\right] \mathrm{d} s \\
= & \int_{0}^{\epsilon} \frac{A M_{0}}{4 s N_{0}^{2}}\left[-2 \frac{s^{n+1}}{\delta} \exp \left(-A^{2} N_{0}^{2} \frac{s^{2 n+2}}{\delta^{2}}\right)+\frac{\sqrt{\pi}}{A N_{0}} \operatorname{erf}\left(A N_{0} \frac{s^{n+1}}{\delta}\right)\right] \mathrm{d} s \\
& +O\left(\epsilon \ln \left[\frac{\epsilon^{n+1}}{\delta}\right]\right) \\
\approx & \frac{-\sqrt{\pi} M_{0}}{4(n+1) N_{0}^{3}} \operatorname{erf}\left(A N_{0} \frac{\epsilon^{n+1}}{\delta}\right)+\frac{\sqrt{\pi} M_{0}}{4(n+1) N_{0}^{3}} \int_{0}^{A N_{0} \epsilon^{n+1} / \delta} \frac{\operatorname{erf}(\xi)}{\xi} \mathrm{d} \xi .
\end{aligned}
$$

From (A9) we have the following relation:

$$
\operatorname{erf}(\xi)=1-\frac{1}{\pi} \int_{0}^{\pi} \exp \left(-\frac{\xi^{2}}{\cos ^{2} \frac{\alpha}{2}}\right) \mathrm{d} \alpha .
$$

Using this relation in (C4) and performing an integration with respect to $\xi$, we obtain

$$
\mathrm{I}_{1} \approx \frac{\sqrt{\pi} M_{0}}{4(n+1) N_{0}^{3}}\left(-\operatorname{erf}\left(A N_{0} \frac{\epsilon^{n+1}}{\delta}\right)+\left[\ln \xi+\frac{1}{2 \pi} \int_{0}^{\pi} \mathrm{E}_{1}\left(\frac{\xi^{2}}{\cos ^{2} \frac{\alpha}{2}}\right) \mathrm{d} \alpha\right]_{\xi=0}^{A N_{0} \epsilon^{n+1} / \delta}\right),
$$

where $\mathrm{E}_{1}$ denotes the exponential integral function. Using $\mathrm{E}_{1}(x) \sim-\gamma-\ln x$ as $x \rightarrow 0$, where $\gamma=0.577$ is the Euler's constant (Abramowitz \& Stegun 1968), we simplify the 


\section{High-Reynolds-number flow past a shear-free cylinder}

limits in (C6) and obtain

$$
\begin{aligned}
\mathrm{I}_{1} \approx & \frac{\sqrt{\pi} M_{0}}{4(n+1) N_{0}^{3}}\left(-\operatorname{erf}\left(A N_{0} \frac{\epsilon^{n+1}}{\delta}\right)+\left[\frac{\gamma}{2}+\ln \left(\frac{2 A N_{0} \epsilon^{n+1}}{\delta}\right)\right.\right. \\
& \left.\left.+\frac{1}{2 \pi} \int_{0}^{\pi} \mathrm{E}_{1}\left(\frac{A^{2} N_{0}^{2} \epsilon^{2 n+2}}{\delta^{2} \cos ^{2} \frac{\alpha}{2}}\right) \mathrm{d} \alpha\right]\right) .
\end{aligned}
$$

Using the fact that $\epsilon^{n+1} \gg \delta, \operatorname{erf}(x) \sim 1-\exp \left(-x^{2}\right) / \sqrt{\pi} x$ and $\mathrm{E}_{1}(x) \sim \exp (-x) / x$ as $x \rightarrow \infty$, we get the leading-order terms in (C7) as follows:

$$
\begin{aligned}
\mathrm{I}_{1}= & \frac{\sqrt{\pi} M_{0}}{4(n+1) N_{0}^{3}}\left(-1+\frac{\gamma}{2}+\ln \left(\frac{2 A N_{0} \epsilon^{n+1}}{\delta}\right)\right)+O\left(\frac{\exp \left(-A^{2} N_{0}^{2} \delta^{-2} \epsilon^{2 n+2}\right)}{\delta^{-1} \epsilon^{n+1}}\right) \\
& +O\left(\epsilon \ln \left(\frac{\epsilon^{n+1}}{\delta}\right)\right) .
\end{aligned}
$$

Next we simplify the second part $\mathrm{I}_{2}$,

$$
\mathrm{I}_{2}=\int_{\epsilon}^{s_{f}} \frac{A M(s)}{4 s N^{2}(s)}\left(\frac{-2 s^{n+1}}{\delta} \exp \left(\frac{-s^{2 n+2} A^{2} N^{2}(s)}{\delta^{2}}\right)+\frac{\sqrt{\pi}}{A N(s)} \operatorname{erf}\left(\frac{s^{n+1} A N(s)}{\delta}\right)\right) \mathrm{d} s .
$$

Using $s^{n+1} / \delta \gg 1$, we expand the integrand in (C9) as follows:

$$
\begin{aligned}
\mathrm{I}_{2} & =\int_{\epsilon}^{s_{f}} \frac{A M(s)}{4 s N^{2}(s)}\left(\frac{\sqrt{\pi}}{A N(s)}-2 \frac{s^{n+1}}{\delta} \exp \left(-A^{2} N^{2}(s) \frac{s^{2 n+2}}{\delta^{2}}\right) \cdots\right) \mathrm{d} s \\
& =\int_{\epsilon}^{s_{f}} \frac{\sqrt{\pi} M(s)}{4 s N^{3}(s)} \mathrm{d} s+O\left(\frac{\epsilon^{n+1} \exp \left(-A^{2} N_{0}^{2} \delta^{-2} \epsilon^{2 n+2}\right)}{\delta}\right) \approx \int_{s=\epsilon}^{s_{f}} \frac{\sqrt{\pi} M(s)}{4 s N^{3}(s)} \mathrm{d} s .
\end{aligned}
$$

Next we apply a singularity subtraction technique to regularize the integrand in $(\mathrm{C} 10)$ as follows:

$$
\mathrm{I}_{2} \approx \int_{s_{0}}^{s_{f}} \frac{\sqrt{\pi} M(s)}{4 s N^{3}(s)} \mathrm{d} s+\int_{\epsilon}^{s_{0}} \frac{\sqrt{\pi}}{4 s}\left(\frac{M(s)}{N^{3}(s)}-\frac{M_{0}}{N_{0}^{3}}\right) \mathrm{d} s+\int_{\epsilon}^{s_{0}} \frac{\sqrt{\pi} M_{0}}{4 s N_{0}^{3}} \mathrm{~d} s .
$$

Here $s_{0} \leqslant s_{f}$ is an $O(1)$ limit that can be set more or less arbitrarily. Since the integrand in the second part remains bounded in the limit $s \rightarrow 0$, the leading-order terms in $\mathrm{I}_{2}$ are readily found, i.e.

$$
\begin{aligned}
\mathrm{I}_{2}= & \int_{s_{0}}^{s_{f}} \frac{\sqrt{\pi} M(s)}{4 s N^{3}(s)} \mathrm{d} s+\int_{0}^{s_{0}} \frac{\sqrt{\pi}}{4 s}\left(\frac{M(s)}{N^{3}(s)}-\frac{M_{0}}{N_{0}^{3}}\right) \mathrm{d} s+\frac{\sqrt{\pi} M_{0}}{4 N_{0}^{3}} \ln \left(\frac{s_{0}}{\epsilon}\right) \\
& +O\left(\frac{\epsilon^{n+1} \exp \left(-A^{2} N_{0}^{2} \delta^{-2} \epsilon^{2 n+2}\right)}{\delta}\right)+O(\epsilon) .
\end{aligned}
$$


Adding the results (C7) and (C12), we deduce the leading-order terms in (C1) as follows:

$$
\begin{aligned}
\mathrm{I} \approx & \int_{s_{0}}^{s_{f}} \frac{\sqrt{\pi} M(s)}{4 s N^{3}(s)} \mathrm{d} s+\int_{0}^{s_{0}} \frac{\sqrt{\pi}}{4 s}\left(\frac{M(s)}{N^{3}(s)}-\frac{M_{0}}{N_{0}^{3}}\right) \mathrm{d} s \\
& +\frac{\sqrt{\pi} M_{0}}{4(n+1) N_{0}^{3}}\left(\frac{\gamma}{2}-1+\ln \left(\frac{2 A N_{0} s_{0}^{n+1}}{\delta}\right)\right) .
\end{aligned}
$$

Thus, our analysis of the leading-order terms separates out the $\delta$-dependence of the integral I. The remaining integrals in (C13) involve non-singular integrands and are therefore amenable to accurate approximation using standard quadrature rules.

Next we show that the integral (4.9) is indeed a special case of the general form (C1) and can therefore be estimated in a manner similar to the one outlined in the foregoing analysis. Expressed in the $\theta$ coordinate, the square of vorticity from the boundary layer region assumes the following form:

$$
\begin{aligned}
\omega_{b l}^{* 2}= & \frac{64}{\pi} \int_{0}^{\pi} \int_{0}^{\pi} y^{* 2} \sin ^{2}\left(\frac{\theta}{2}\right) \cos ^{2}\left(\frac{\theta}{2}\right) \exp \left(-y^{* 2}\left[\frac{\sin ^{2}\left(\frac{\theta}{2}\right)}{\cos ^{2}\left(\frac{\alpha_{1}}{2}\right)}+\frac{\sin ^{2}\left(\frac{\theta}{2}\right)}{\cos ^{2}\left(\frac{\alpha_{2}}{2}\right)}\right]\right) \\
& \times \tan ^{2}\left(\frac{\alpha_{1}}{2}\right) \tan ^{2}\left(\frac{\alpha_{2}}{2}\right) \sqrt{1-\sin ^{2}\left(\frac{\alpha_{1}}{2}\right) \cos ^{2}\left(\frac{\theta}{2}\right)} \\
& \sqrt{1-\sin ^{2}\left(\frac{\alpha_{2}}{2}\right) \cos ^{2}\left(\frac{\theta}{2}\right)} \mathrm{d} \alpha_{1} \mathrm{~d} \alpha_{2} .
\end{aligned}
$$

From the above relationship, we can recast the boundary layer contribution to the vorticity-squared integral in (4.9) as follows:

$$
\begin{aligned}
& \int_{\theta=0}^{\pi} \int_{y^{*}=0}^{(1 / \delta)(\theta / A)^{1 / b}} \omega_{b l}^{* 2} \mathrm{~d} y^{*} \mathrm{~d} \theta \\
& \quad=\frac{64}{\pi} \int_{0}^{\pi} \int_{0}^{\pi} \tan ^{2}\left(\frac{\alpha_{1}}{2}\right) \tan ^{2}\left(\frac{\alpha_{2}}{2}\right) \mathrm{I}\left(1 / A^{1 / b}, 1 / b, \pi, \delta, M(\theta), N(\theta)\right) \mathrm{d} \alpha_{1} \mathrm{~d} \alpha_{2} .
\end{aligned}
$$

Here

$$
\begin{aligned}
M(\theta) & =\frac{\sin ^{2}\left(\frac{\theta}{2}\right)}{\theta^{2}} \cos ^{2}\left(\frac{\theta}{2}\right) \sqrt{1-\sin ^{2}\left(\frac{\alpha_{1}}{2}\right) \cos ^{2}\left(\frac{\theta}{2}\right)} \sqrt{1-\sin ^{2}\left(\frac{\alpha_{2}}{2}\right) \cos ^{2}\left(\frac{\theta}{2}\right)} \\
\text { and } N(\theta) & =\frac{\sin \left(\frac{\theta}{2}\right)}{\theta} \sqrt{\frac{1}{\cos ^{2}\left(\frac{\alpha_{1}}{2}\right)}+\frac{1}{\cos ^{2}\left(\frac{\alpha_{2}}{2}\right)}} .
\end{aligned}
$$




\section{High-Reynolds-number flow past a shear-free cylinder}

Likewise, the wake contribution to the vorticity-squared integral in (4.9) can be expressed in the general form $(\mathrm{C} 1)$, i.e.

$$
\begin{aligned}
& \int_{x=0}^{\infty} \int_{z^{*}=0}^{A x^{b} / \delta} \omega_{w}^{* 2} \mathrm{~d} z^{*} \mathrm{~d} x \\
& \quad=\frac{2}{\pi} \int_{-2}^{2} \int_{-2}^{2} \sqrt{4-\kappa_{1}^{2}} \sqrt{4-\kappa_{2}^{2}} \mathrm{I}(A, b, \infty, \delta, M(x), N(x)) \mathrm{d} \kappa_{1} \mathrm{~d} \kappa_{2},
\end{aligned}
$$

where

$$
\begin{aligned}
M(x) & =\frac{\left(1-\frac{1}{(x+1)^{2}}\right)^{2}}{2 x^{2}\left(x+1+\frac{1}{x+1}-\kappa_{1}\right)^{3 / 2}\left(x+1+\frac{1}{x+1}-\kappa_{2}\right)^{3 / 2}} \\
\text { and } \quad N(x) & =\frac{\left(1-\frac{1}{(x+1)^{2}}\right)}{2 x} \sqrt{\frac{1}{x+1+\frac{1}{x+1}-\kappa_{1}}+\frac{1}{x+1+\frac{1}{x+1}-\kappa_{2}}} .
\end{aligned}
$$

Making use of the foregoing analysis in the derivation of the leading-order terms and in the quadrature-based numerical approximation of the remaining regularized integrals in (C15) and (C17), we readily establish the estimate given by (4.9).

\section{REFERENCES}

Abramowitz, M. \& Stegun, I.A. 1968 Handbook of Mathematical Functions: With Formulas, Graphs, and Mathematical Tables. Dover.

ARAKERI, J.H. \& SHUKLA, R.K. 2013 A unified view of energetic efficiency in active drag reduction, thrust generation and self-propulsion through a loss coefficient with some applications. J. Fluid Struct. 41, 22-32.

BAtChelor, G.K. 2000 An Introduction to Fluid Dynamics. Cambridge University Press.

BocQuet, L. \& LaUga, E. 2011 A smooth future? Nat. Mater. 10, 334-337.

CRAIG, J.W. 1991 A new, simple and exact result for calculating the probability of error for two-dimensional signal constellations. In Military Communications Conference, 1991. MILCOM'91, Conference Record, Military Communications in a Changing World, pp. 571-575. IEEE.

HaAse, S.A., Chapman, S.J., Tsai, P.A., Lohse, D. \& Lammertink, R.G.H. 2015 The Graetz-Nusselt problem extended to continuum flows with finite slip. J. Fluid Mech. 764, R3.

HaAse, S.A. \& LAmmertink, R.G.H. 2016 Heat and mass transfer over slippery, superhydrophobic surfaces. Phys. Fluids 28, 042002.

HARPER, J.F. 1963 On boundary layers in two-dimensional flow with vorticity. J. Fluid Mech. 17 (1), 141-153.

HARPER, J.F. 1972 The motion of bubbles and drops through liquids. Adv. Appl. Mech. 12, 59-129.

HARPER, J.F. \& MOORE, D.W. 1968 The motion of a spherical liquid drop at high Reynolds number. J. Fluid Mech. 32 (2), 367-391.

Hinch, E.J. 1991 Perturbation Methods. Cambridge University Press.

Hugues, S. \& RANDRiamampianina, A. 1998 An improved projection scheme applied to pseudospectral methods for the incompressible Navier-Stokes equations. Intl J. Numer. Meth. Fluids 28 (3), 501-521.

Joseph, D.D., FunADA, T. \& WANG, J. 2007 Potential Flows of Viscous and Viscoelastic Fluids. Cambridge University Press.

KANG, I.S. \& LEAL, L.G. 1988 The drag coefficient for a spherical bubble in a uniform streaming flow. Phys. Fluids 31 (2), 233-237.

Karatay, E., Haase, A.S., Visser, C.W., Sun, C., Lohse, D., Tsai, P.A. \& Lammertink, R.G.H. 2013 Control of slippage with tunable bubble mattresses. Proc. Natl Acad. Sci. 110 (21), 8422-8426.

VON KÁRMÁN, T. 1911 Über den mechanismus des widerstandes, den ein bewegter körper in einer flüssigkeit erfährt. Nachr. Ges. Wiss. Göttingen, Math.-Phys. Kl. 1911, 509-517. 


\section{A. Kumar, N.M.A. Rehman, P. Giri and R.K. Shukla}

LAmb, H. 1932 Hydrodynamics. Cambridge University Press.

LEAL, L.G. 1989 Vorticity transport and wake structure for bluff bodies at finite Reynolds number. Phys. Fluids 1 (1), 124-131.

Leal, L.G. 2007 Advanced Transport Phenomena: Fluid Mechanics and Convective Transport Processes. Cambridge University Press.

Legendre, D., Lauga, E. \& MAgnaudet, J. 2009 Influence of slip on the dynamics of two-dimensional wakes. J. Fluid Mech. 633, 437-447.

LEVICH, V.G. 1949 The motion of bubbles at high Reynolds numbers. Zh. Eksp. Teor. Fiz. 19 (18), 436f.

Li, D., Li, S., Xue, Y., Yang, Y., Su, W., Xia, Z., Shi, Y., Lin, H. \& Duan, H. 2014 The effect of slip distribution on flow past a circular cylinder. J. Fluid Struct. 51, 211-224.

MAGNAUDET, J. \& EAMES, I. 2000 The motion of high-Reynolds-number bubbles in inhomogeneous flows. Annu. Rev. Fluid Mech. 32, 659-708.

MichaELIDES, E.E. 2003 Hydrodynamic force and heat/mass transfer from particles, bubbles, and drops-The Freeman scholar lecture. Trans. ASME J. Fluids Engng 125, 209-238.

MoOre, D.W. 1963 The boundary layer on a spherical gas bubble. J. Fluid Mech. 16 (2), 161-176.

Moore, D.W. 1965 The velocity of rise of distorted gas bubbles in a liquid of small viscosity. J. Fluid Mech. 23 (4), 749-766.

Muralidhar, P., Ferrer, N., Daniello, R. \& Rothstein, J.P. 2011 Influence of slip on the flow past superhydrophobic circular cylinders. J. Fluid Mech. 680, 459-476.

Ou, J., PEROT, B. \& Rothstein, J.P. 2004 Laminar drag reduction in microchannels using ultrahydrophobic surfaces. Phys. Fluids 16 (12), 4635-4643.

Rehman, N.M.A., Kumar, A. \& ShukLa, R.K 2017 Influence of hydrodynamic slip on convective transport in flow past a circular cylinder. Theor. Comput. Fluid Dyn. 31, 251-280.

RothStein, J.P. 2010 Slip on superhydrophobic surfaces. Annu. Rev. Fluid Mech. 42, 89-109.

SChlichting, H. \& Gersten, K. 2003 Boundary Layer Theory. Springer.

SEO, I.W. \& SonG, C.G. 2012 Numerical simulation of laminar flow past a circular cylinder with slip conditions. Intl J. Numer. Meth. Fluids 68 (12), 1538-1560.

SHUKLA, R.K. \& ARAKERI, J.H. 2013 Minimum power consumption for drag reduction on a circular cylinder by tangential surface motion. J. Fluid Mech. 715, 597-641.

ShUKLA, R.K., TATINENI, M. \& ZHONG, X. 2007 Very high-order compact finite difference schemes on non-uniform grids for incompressible Navier-Stokes equations. J. Comput. Phys. 224 (2), 1064-1094.

ShuKLA, R.K \& ZHONG, X. 2005 Derivation of high-order compact finite difference schemes for non-uniform grid using polynomial interpolation. J. Comput. Phys. 204 (2), 404-429.

Sooraj, P., Ramagya, M.S., Khan, M.H., Sharma, A. \& Agrawal, A. 2020 Effect of superhydrophobicity on the flow past a circular cylinder in various flow regimes. J. Fluid Mech. 897, A21.

Strouhal, V. 1878 Ueber eine besondere art der tonerregung. Ann. Phys. Chem. (New series) 5, 216-251.

VAN Dy Ke, M. 1975 Perturbation Methods in Fluid Mechanics. The Parabolic Press.

Williamson, C.H.K. 1996 Vortex dynamics in the cylinder wake. Annu. Rev. Fluid Mech. 28, 477-539.

XIONG, Y.L. \& YANG, D. 2017 Influence of slip on the three-dimensional instability of flow past an elongated superhydrophobic bluff body. J. Fluid Mech. 814, 69-94.

You, D. \& MoIN, P. 2007 Effects of hydrophobic surfaces on the drag and lift of a circular cylinder. Phys. Fluids 19 (8), 081701. 